\title{
THE COMMUTATIVE INVERSE SEMIGROUP OF PARTIAL ABELIAN EXTENSIONS
}

\author{
DIRCEU BAGIO, ANDRÉS CAÑAS, VÍCTOR MARÍN, ANTONIO PAQUES, \\ AND HÉCTOR PINEDO
}

\begin{abstract}
This paper is a new contribution to the partial Galois theory of groups. First, given a unital partial action $\alpha_{G}$ of a finite group $G$ on an algebra $S$ such that $S$ is an $\alpha_{G}$-partial Galois extension of $S^{\alpha_{G}}$ and a normal subgroup $H$ of $G$, we prove that $\alpha_{G}$ induces a unital partial action $\alpha_{G / H}$ of $G / H$ on the subalgebra of invariants $S^{\alpha_{H}}$ of $S$ such that $S^{\alpha_{H}}$ is an $\alpha_{G / H}$-partial Galois extension of $S^{\alpha_{G}}$. Second, assuming that $G$ is abelian, we construct a commutative inverse semigroup $T_{\text {par }}(G, R)$, whose elements are equivalence classes of $\alpha_{G}$-partial abelian extensions of a commutative algebra $R$. We also prove that there exists a group isomorphism between $T_{p a r}(G, R) / \rho$ and $T(G, R)$, where $\rho$ is a congruence on $T_{\text {par }}(G, R)$ and $T(G, R)$ is the classical Harrison group of the $G$-isomorphism classes of the abelian extensions of $R$. It is shown that the study of $T_{\text {par }}(G, R)$ reduces to the case where $G$ is cyclic. The set of idempotents of $T_{\text {par }}(G, R)$ is also investigated.
\end{abstract}

\section{INTRODUCTION}

In the 1960's, M. Auslander and O. Goldman introduced in [1] the notion of Galois extension for commutative rings. After that, S. U. Chase, D. K. Harrison and A. Rosenberg developed in [2] a Galois theory for commutative rings extending the classical theory over fields. One of the main results in [2] is Theorem 2.3 which has two parts as described in the sequel. Let $S \supset R$ be a Galois extension of commutative rings with a finite group $G$ as Galois group (shortly, a $G$-extension of $R$ ). The first part establishes a bijective correspondence between all the subgroups of $G$ and the subrings of $S$ which are $G$-strong and separable as $R$-algebras. In the second part, it was shown that if $H$ is a normal subgroup of $G$ then the subring $S^{H}$ of $S$ of the invariants by the action of $H$ is a $G / H$-extension of $R$.

Given a commutative ring extension $T \supset R$ and a finite group $G$ we say that $T$ is an abelian $G$-extension of $R$ if $T$ is a $G$-extension of $R$ and $G$ is abelian. Given a finite abelian group $G$ and a commutative algebra $R$, D. K. Harrison constructed in [6] the group $\mathrm{T}(G, R)$, which is called the Harrison group of the abelian $G$-extensions of $R$. The elements of $\mathrm{T}(G, R)$ are $G$-isomorphism classes of abelian $G$-extensions of $R$. The binary operation in $\mathrm{T}(G, R)$ is defined in the following way. Let $T$ and $T^{\prime}$ be abelian $G$-extensions of $R$. Thus, $T \otimes_{R} T^{\prime}$ is an abelian $(G \times G)$-extension of $R \otimes_{R} R \simeq R$, where the action is given by: $(g, h)((a \otimes b))=g(a) \otimes h(b)$, for all $g, h \in G, a \in T$ and

Date: September 29, 2020.

Mathematics Subject Classification: Primary 13B05, 13A50. Secondary 20M14, 20M18.

Key words and phrases: Partial Galois abelian extension, Harrison group, inverse semigroup. 
$b \in T^{\prime}$. Consider the subgroup $\delta G=\left\{\left(g, g^{-1}\right): g \in G\right\}$ of $G \times G$. By the second part of Theorem 2.3 of [2], $\left(T \otimes_{R} T^{\prime}\right)^{\delta G}$ is an abelian $((G \times G) / \delta G) \simeq G$-extension of $R$ and whence its equivalence class is an element of $\mathrm{T}(G, R)$. Therefore, we have an operation on $\mathrm{T}(G, R)$. In [6], it was proved that $\mathrm{T}(G, R)$ is an abelian group.

The purpose of this paper is to explore such a construction due to D. K. Harrison in the context of partial Galois extensions. For this, we need first to extend the second part of Theorem 2.3 of [2] to the context of partial group actions.

In [4], a Galois theory of commutative rings for partial group actions was developed. The results proved in [4] generalize many results of [2]. Particularly, Theorem 5.1 of [4] is the version of the first part of Theorem 2.3 of [2] in the context of partial actions. However, a version for the second part of such a theorem was not considered in [4]. In our first main result, which is presented below, we provide this.

Theorem 3.1. Let $R, S$ be algebras, $G$ a finite group, $\alpha_{G}$ a unital partial action of $G$ on $S$ such that $S$ is a partial $\alpha_{G^{-}}$extension of $R$ and $H$ a normal subgroup of $G$. Then $\alpha_{G}$ induces a unital partial action $\alpha_{G / H}$ of the quotient group $G / H$ on the subalgebra of invariants $S^{\alpha_{H}}$ such that $S^{\alpha_{H}}$ is a partial $\alpha_{G / H^{-}}$extension of $R$ and $\left(S^{\alpha_{H}}\right)^{\alpha_{G / H}}=R$.

The above theorem allows to reproduce the Harrison's construction for partial actions. Given a finite abelian group $G$ and a commutative algebra $R$, we consider the set $\mathrm{T}_{\text {par }}(G, R)$ of the $G$-isomorphism classes of (unital) partial abelian $\alpha_{G}$-extensions of $R$. Let $\alpha_{G}$ and $\alpha_{G}^{\prime}$ be unital partial actions of $G$ on the algebras $S$ and $S^{\prime}$ respectively. Suppose that $S$ (resp. $S^{\prime}$ ) is a partial abelian $\alpha_{G^{-}}$extension (resp. $\alpha_{G}^{\prime}$-extension) of $R$. By Proposition 2.9 of [5], $\alpha_{G \times G}=\alpha_{G} \otimes \alpha_{G}^{\prime}$ is a unital partial action of $G \times G$ on $S \otimes_{R} S^{\prime}$, where $\alpha_{(g, h)}:=\alpha_{g} \otimes \alpha_{h}^{\prime}$, for all $g, h \in G$. Moreover, $S \otimes_{R} S^{\prime}$ is a partial abelian $\left(\alpha_{G} \otimes \alpha_{G}^{\prime}\right)$-extension of $R \otimes_{R} R \simeq R$. Thus, by Theorem $3.1\left(S \otimes_{R} S^{\prime}\right)^{\alpha_{\delta G}}$ is a partial abelian $\alpha_{(G \times G) / \delta_{G}}$-extension of $R$. Hence, we have an operation on $\mathrm{T}_{p a r}(G, R)$ given by

$$
\left\lfloor S, \alpha_{G}\right\rfloor *_{\text {par }}\left\lfloor S^{\prime}, \alpha_{G}^{\prime}\right\rfloor=\left\lfloor\left(S \otimes_{R} S^{\prime}\right)^{\alpha_{\delta G}}, \alpha_{(G \times G) / \delta_{G}}\right\rfloor,
$$

where $\lfloor L, \theta\rfloor$ denotes the class of $G$-isomorphism of a partial abelian $\theta$-extension $L$ of $R$. We will show that $*_{\text {par }}$ is a well-defined associative operation on $T_{p a r}(G, R)$. In fact, we have the following.

Theorem 5.1. Let $G$ be a finite abelian group and $R$ a commutative algebra. Then $\left(T_{\text {par }}(G, R), *_{\text {par }}\right)$ is a commutative inverse semigroup.

We shall establish a relation between $\mathrm{T}_{\text {par }}(G, R)$ and a Harrison group. Indeed, consider $\left\lfloor S, \alpha_{G}\right\rfloor \in \mathrm{T}_{\text {par }}(G, R)$ and let $\left(T, \beta_{G}\right)$ be the globalization of $\left(S, \alpha_{G}\right)$. By Theorem 3.3 of [4], $T$ is a (global) $G$-extension of $A=T^{\beta_{G}}$ and consequently its equivalence class $\left[T, \beta_{G}\right]$ is an element of $\mathrm{T}(G, A)$. Using Theorem 4.3, we will prove that the association $\lfloor S, \alpha\rfloor \mapsto[T, \beta]$ is a well-defined semigroup homomorphism and we have the following result.

Theorem 5.3. Let $R$ be an algebra, $\pi: \mathrm{T}_{\text {par }}(G, R) \rightarrow \mathrm{T}(G, A)$ the semigroup homomorphism defined by $\pi(\lfloor S, \alpha\rfloor)=[T, \beta]$ and $\rho=\operatorname{ker} \pi$. Then, the canonical map induced by $\pi$ from $\mathrm{T}_{\text {par }}(G, R) / \rho$ to $\mathrm{T}(G, A)$ is an isomorphism of groups.

Commutative inverse semigroups are strong semilattices of abelian groups. In particular, if $\mathcal{I}$ is a commutative inverse semigroup then $\mathcal{I}=\bigcup_{e \in E(\mathcal{I})} \mathcal{I}_{e}$ where $E(\mathcal{I})$ is the 
semillatice of the idempotents of $\mathcal{I}$ and $\mathcal{I}_{e}$ is a group, for all $e \in E(\mathcal{I})$. In the last section of this paper we study the idempotents of $\mathrm{T}_{\text {par }}(G, R)$.

The paper is organized as follows. The basic notions and results that we used throughout the paper are presented in Section 2. In Section 3, we will prove Theorem 3.1. Following ideas from [4], in Section 4 we study partial Galois extensions which are $G$ isomorphic. In Section 5, we prove that $\mathrm{T}_{p a r}(G, R)$ is a commutative inverse semigroup. Some aspects of the structure of $\mathrm{T}_{\text {par }}(G, R)$ are dealt with in Section 6 .

Conventions. Throughout the paper, $k$ will denote an associative and commutative ring with identity element. All algebras are $k$-algebras and they are considered associative with identity element. Each algebra homomorphism is unitary, that is, it preserves identity elements. Extensions of algebras have the same identity element. If $S$ and $T$ are extensions of an algebra $R$ then $S \otimes T$ means $S \otimes_{R} T$. As usual, the annihilator of a central element $x$ of an algebra $T$ will be denoted by ann $(x)$, i. e. $\operatorname{ann}(x)=\{t \in T: t x=0\}$. Similarly, if $T$ is an algebra extension of $R$ and $x$ is a central element of $T$ then $\operatorname{ann}_{R}(x):=\{r \in R: r x=0\}$. We will denote the identity element of a group $G$ by 1 .

\section{Preliminaries}

In this section we present the background that will be used in the paper. The references used here are [3] and [4].

2.1. Partial action of groups. A partial action of a group $G$ on a $k$-algebra $S$ is a family of pairs $\alpha_{G}=\left(S_{g}, \alpha_{g}\right)_{g \in G}$ that satisfies:

(P1) for each $g \in G, S_{g}$ is an ideal of $S$ and $\alpha_{g}: S_{g^{-1}} \rightarrow S_{g}$ is a $k$-algebra isomorphism,

(P2) $S_{1}=S$ and $\alpha_{1}=\operatorname{id}_{S}$,

(P3) $\alpha_{g}\left(S_{g^{-1}} \cap S_{h}\right)=S_{g} \cap S_{g h}$, for all $g, h \in G$,

(P4) $\alpha_{g} \circ \alpha_{h}(x)=\alpha_{g h}(x)$, for all $x \in \alpha_{h^{-1}}\left(S_{h} \cap S_{g^{-1}}\right)$ and $g, h \in G$.

The partial action $\alpha_{G}$ is called unital if every ideal $S_{g}$ is unital, that is, there exists a central idempotent $1_{g}$ in $S$ such that $S_{g}=S 1_{g}$. Notice that the conditions (P3) and (P4) imply that $\alpha_{g h}$ is an extension of $\alpha_{g} \circ \alpha_{h}$, for every $g, h \in G$. For a subgroup $H$ of $G$, the partial action $\alpha_{H}$ of $H$ on $S$ is obtained by restriction of $\alpha_{G}$, i. e. $\alpha_{H}=\left(S_{h}, \alpha_{h}\right)_{h \in H}$. Sometimes, we will write $\left(S, \alpha_{G}\right)$ to denote a partial action of $G$ on $S$.

A partial action $\alpha_{G}=\left(S_{g}, \alpha_{g}\right)_{g \in G}$ is said global if $S_{g}=S$, for all $g \in G$. Global actions of a group $G$ on a $k$-algebra $T$ induce, by restriction, partial actions on any ideal $S$ of $T$. Indeed, given a global action $\beta_{G}=\left(T_{g}, \beta_{g}\right)_{g \in G}$ of $G$ on $T$ we consider the ideals $S_{g}=S \cap \beta_{g}(S)$ of $S$ and the $k$-algebra isomorphisms $\alpha_{g}=\left.\beta_{g}\right|_{S_{g-1}}$. Then $\alpha_{G}=\left(S_{g}, \alpha_{g}\right)_{g \in G}$ is a partial action of $G$ on $S$. We are interested in partial actions that are globalizable, that is, those that can be obtained as restriction of global actions.

A globalization of a partial action $\alpha_{G}=\left(S_{g}, \alpha_{g}\right)_{g \in G}$ of a group $G$ on a $k$-algebra $S$ is a global action $\beta_{G}=\left(T_{g}, \beta_{g}\right)_{g \in G}$ of $G$ on a $k$-algebra $T$ with a monomorphism $\varphi: S \rightarrow T$ of $k$-algebras such that:

(G1) $\varphi(S)$ is an ideal of $T$, 
(G2) $\varphi\left(S_{g}\right)=\varphi(S) \cap \beta_{g}(\varphi(S))$, for all $g \in G$,

(G3) $\beta_{g}(\varphi(x))=\varphi\left(\alpha_{g}(x)\right)$, for all $x \in S_{g^{-1}}$,

(G4) $T=\sum_{g \in G} \beta_{g}(\varphi(S))$.

If $\alpha_{G}$ admits a globalization we say that $\alpha$ is globalizable and, in this case, its globalization is unique, up to isomorphism. Also, Theorem 4.5 of [3] implies that unital partial actions admit globalization. More details related to globalization can be seen in [3].

From now on we assume that $G$ is a finite group, $\alpha_{G}=\left(S_{g}, \alpha_{g}\right)_{g \in G}$ is a unital partial action of $G$ on a $k$-algebra $S$ such that $S_{g}=S 1_{g}$, for all $g \in G$, and $\beta_{G}=\left(T_{g}, \beta_{g}\right)_{g \in G}$ is a global action of $G$ on a $k$-algebra $T$ which is the globalization of $\alpha_{G}$. In order to simplify the notation, we assume that the injective morphism $\varphi$ in the definition of globalization is the inclusion map from $S$ to $T$, that is, $S$ is an ideal of $T$. Notice that $1_{S}$ is a central idempotent of $T$ and $S=T 1_{S}$. Moreover, it was proved in [4, p.79] that

(1) $1_{g}=\beta_{g}\left(1_{S}\right) 1_{S}, \quad \alpha_{g}\left(s 1_{g^{-1}}\right)=\beta_{g}(s) 1_{S}, \quad \alpha_{g}\left(\alpha_{h}\left(s 1_{h^{-1}}\right) 1_{g^{-1}}\right)=\alpha_{g h}\left(s 1_{(g h)^{-1}}\right) 1_{g}$,

for all $g, h \in G$ and $s \in S$. Let $H=\left\{h_{1}=1, h_{2}, \ldots, h_{m}\right\}$ be a subgroup of $G$. It was defined in $\left[4\right.$, p.79] the map $\psi_{H}: T \rightarrow T$ by

$$
\psi_{H}(t)=\sum_{1 \leq l \leq m} \sum_{i_{1}<\cdots<i_{l}}(-1)^{l+1} \beta_{h_{i_{1}}}\left(1_{S}\right) \beta_{h_{i_{2}}}\left(1_{S}\right) \cdots \beta_{h_{i_{l}}}\left(1_{S}\right) \beta_{h_{i_{l}}}(t), \quad t \in T .
$$

This map can be rewritten as

$$
\psi_{H}(t)=\sum_{i=1}^{m} \beta_{h_{i}}(t) e_{i}, \quad \text { for all } t \in T,
$$

where $e_{i}$ 's are the following idempotents of $T$ :

$$
e_{1}=1_{S}, \quad e_{i}=\left(1_{T}-1_{S}\right)\left(1_{T}-\beta_{h_{2}}\left(1_{S}\right)\right) \cdots\left(1_{T}-\beta_{h_{i-1}}\left(1_{S}\right)\right) \beta_{h_{i}}\left(1_{S}\right), \quad 2 \leq i \leq m .
$$

Since $1_{S}$ is a central idempotent of $T$, given $2 \leq i \leq m$ we have

$$
\begin{aligned}
\beta_{g}\left(e_{i}\right) 1_{S} & =\left(1_{T}-\beta_{g}\left(1_{S}\right)\right)\left(1_{T}-\beta_{g h_{2}}\left(1_{S}\right)\right) \cdots\left(1_{T}-\beta_{g h_{i-1}}\left(1_{S}\right)\right) \beta_{g h_{i}}\left(1_{S}\right) 1_{S} \\
& =\left(1_{S}-\beta_{g}\left(1_{S}\right) 1_{S}\right)\left(1_{S}-\beta_{g h_{2}}\left(1_{S}\right) 1_{S}\right) \cdots\left(1_{S}-\beta_{g h_{i-1}}\left(1_{S}\right) 1_{S}\right) \beta_{g h_{i}}\left(1_{S}\right) 1_{S} \\
& \stackrel{(1)}{=}\left(1_{S}-1_{g}\right)\left(1_{S}-1_{g h_{2}}\right) \cdots\left(1_{S}-1_{g h_{i-1}}\right) 1_{g h_{i}} .
\end{aligned}
$$

Thus

$$
\beta_{g}\left(e_{1}\right) 1_{S}=1_{g} \text { and } \beta_{g}\left(e_{i}\right) 1_{S}=\prod_{j=2}^{i}\left(1_{S}-1_{g h_{j-1}}\right) 1_{g h_{i}}, \quad 2 \leq i \leq m .
$$

We recall from [4] that $S^{\alpha_{G}}:=\left\{s \in S: \alpha_{g}\left(s 1_{g^{-1}}\right)=s 1_{g}\right.$, for all $\left.g \in G\right\}$ is called the subalgebra of invariants of $S$ under $\alpha_{G}$. If $\alpha_{G}$ is global then $S^{\alpha_{G}}$ is the classical subalgebra of invariants, i. e. $S^{\alpha_{G}}=S^{G}=\left\{x \in S: \alpha_{g}(s)=s\right.$, for all $\left.g \in G\right\}$.

We shall denote by $e_{H}$ the image of $1_{S}$ by $\psi_{H}$, that is,

$$
e_{H}=\psi_{H}\left(1_{S}\right) \text {. }
$$

The element $e_{H} \in T$ will be useful in Section 3. Some properties of the map $\psi_{H}$ and of the element $e_{H}$ are given in the next. 
Proposition 2.1. Let $H=\left\{h_{1}=1, h_{2}, \ldots, h_{m}\right\}$ be a subgroup of $G$ and $\psi_{H}: T \rightarrow T$ the map defined in (2). Then:

(i) $\psi_{H}$ is a k-algebra homomorphism,

(ii) $\psi_{H}$ is left and right $T^{\beta_{H}}$-linear map,

(iii) the restriction $\left.\psi_{H}\right|_{S}$ to $S$ is injective,

(iv) $e_{H}$ is a central idempotent of $T$,

(v) $\psi_{H}\left(S^{\alpha_{H}}\right) \subset T^{\beta_{H}}$,

(vi) the restriction of $\psi_{H}$ to $S^{\alpha_{H}}$ is a k-algebra isomorphism from $S^{\alpha_{H}}$ onto $T^{\beta_{H}} e_{H}$ whose inverse is the multiplication by $1_{S}$. In particular $T^{\beta_{H}} 1_{S}=S^{\alpha_{H}}$.

Proof. Notice that (i) is immediate because each $\beta_{g}, g \in G$, is a $k$-algebra homomorphism and $\left\{e_{i}: 1 \leq i \leq m\right\}$ is a set of orthogonal idempotents of $T$. Item (ii) is clear. For (iii), take $s \in S$ and observe that

$$
\psi_{H}(s) 1_{S}=\sum_{i=1}^{m} \beta_{h_{i}}(s) e_{i} 1_{S}=\beta_{1}(s) 1_{S}=s
$$

because $e_{i} 1_{S}=0$, for all $2 \leq i \leq m$. Thus, (iii) follows. Since $\left\{e_{i}: 1 \leq i \leq m\right\}$ is a set of central orthogonal idempotents of $T$ and $\psi_{H}\left(1_{S}\right)=e_{1}+\cdots+e_{m}$, item (iv) follows.

For $(\mathrm{v})$, we need to show that $\beta_{h}\left(\psi_{H}(s)\right)=\psi_{H}(s)$, for all $s \in S^{\alpha_{H}}$ and $h \in H$. Thus, it is enough to check that $\beta_{h}\left(\psi_{H}(s)\right)$ gives us a permutation of the terms that appear in the sum of $\psi_{H}(s)$ given in (2). First, observe that

$$
\begin{aligned}
\beta_{h_{i}}\left(1_{S}\right) \beta_{h_{j}}(s) & =\beta_{h_{j}}\left(\beta_{h_{j}^{-1} h_{i}}\left(1_{S}\right) s\right)=\beta_{h_{j}}\left(\beta_{h_{j}^{-1} h_{i}}\left(1_{S}\right) s 1_{S}\right) \\
& \stackrel{(1)}{=} \beta_{h_{j}}\left(1_{h_{j}^{-1} h_{i}} s\right) \stackrel{(\star)}{=} \beta_{h_{j}}\left(\beta_{h_{j}^{-1} h_{i}}(s) 1_{S}\right) \\
& =\beta_{h_{i}}(s) \beta_{h_{j}}\left(1_{S}\right) \stackrel{(\star \star)}{=} \beta_{h_{j}}\left(1_{S}\right) \beta_{h_{i}}(s),
\end{aligned}
$$

where $(\star)$ follows because $\beta_{h}(s) 1_{S} \stackrel{(1)}{=} \alpha_{h}\left(s 1_{h^{-1}}\right)=s 1_{h}$ and $(* *)$ follows using the fact that $\beta_{h}\left(1_{S}\right)$ is a central element of $T$, for all $h \in H$. Now, for $1 \leq l \leq n$, consider $i_{1}<\cdots<i_{l}$ and note that

$$
\begin{aligned}
\beta_{h}\left(\beta_{h_{i_{1}}}\left(1_{S}\right) \cdots \beta_{h_{i_{l-1}}}\left(1_{S}\right) \beta_{h_{i_{l}}}(s)\right) & =\beta_{h h_{i_{1}}}\left(1_{S}\right) \cdots \beta_{h h_{i_{l-1}}}\left(1_{S}\right) \beta_{h h_{i_{l}}}(s) \\
& =\beta_{h_{j_{1}}}\left(1_{S}\right) \cdots \beta_{h_{j_{l-1}}}\left(1_{S}\right) \beta_{h_{j_{l}}}(s),
\end{aligned}
$$

where $h_{j_{k}}=h h_{i_{k}}$ for all $1 \leq k \leq l$. Since each $\beta_{h}\left(1_{S}\right), h \in H$, is a central element of $T$, we can rearrange $\beta_{h_{j_{1}}}\left(1_{S}\right), \ldots, \beta_{h_{j_{l-1}}}\left(1_{S}\right)$ such that $j_{1}<\cdots<j_{l-1}$. If $j_{l-1}<j_{l}$, there is nothing to do. Otherwise, as we showed above, $\beta_{h_{l-1}}\left(1_{S}\right) \beta_{h_{l}}(s)=\beta_{h_{l}}\left(1_{S}\right) \beta_{h_{l-1}}(s)$. Thus, $\beta_{h}\left(\beta_{h_{i_{1}}}\left(1_{S}\right) \cdots \beta_{h_{i_{l-1}}}\left(1_{S}\right) \beta_{h_{i_{l}}}(s)\right)$ appears in the sum of $\psi_{H}(s)$ and the result follows.

Finally for (vi), notice that by (iv) and (v) we have $\psi_{H}\left(S^{\alpha_{H}}\right)=\psi_{H}\left(S^{\alpha_{H}} 1_{S}\right) \subset T^{\beta_{H}} e_{H}$. Hence, by (1), we conclude that $\psi_{H}: S^{\alpha_{H}} \rightarrow T^{\beta_{H}} e_{H}$ is a $k$-algebra homomorphism. Also, 
if $x \in T^{\beta_{H}}$ then

$$
\begin{aligned}
\alpha_{h}\left(\left(x 1_{S}\right) 1_{h^{-1}}\right) & =\beta_{h}\left(\left(x 1_{S}\right) 1_{h^{-1}}\right) \stackrel{(1)}{=} \beta_{h}\left(x 1_{S} \beta_{h^{-1}}\left(1_{S}\right)\right) \\
& =\beta_{h}(x) \beta_{h}\left(1_{S}\right) 1_{S} \stackrel{(1)}{=} x 1_{h}=\left(x 1_{S}\right) 1_{h},
\end{aligned}
$$

for all $h \in H$. Hence, $x 1_{S} \in S^{\alpha_{H}}$. Thus, $\varphi: T^{\beta_{H}} e_{H} \rightarrow S^{\alpha_{H}}$, given by $\varphi(a)=a 1_{S}$ for all $a \in T^{\beta_{H}} e_{H}$, is a well-defined $k$-algebra homomorphism because $e_{H} 1_{S}=1_{S}$. Clearly, $\varphi\left(\psi_{H}(s)\right)=s$ and $\psi_{H}\left(\varphi\left(x e_{H}\right)\right)=x e_{H}$, for all $s \in S^{\alpha_{H}}$ and $x \in T^{\beta_{H}}$.

\section{Partial actions of quotient groups}

From now on all algebras will be commutative. Consider an algebra $R$ and a unital partial action $\left(S, \alpha_{G}\right)$ of $G$ on an algebra $S$. According [4], $S$ is an $\alpha_{G}$-partial Galois extension of $R$ (shortly, a partial $\alpha_{G^{-}}$extension of $R$ ) if $R \simeq S^{\alpha_{G}}$ (as $k$-algebras) and there exist $m \in \mathbb{N}$ and elements $x_{i}, y_{i} \in S, 1 \leq i \leq m$, such that

$$
\sum_{i=1}^{m} x_{i} \alpha_{g}\left(y_{i} 1_{g^{-1}}\right)=\delta_{1, g}, \text { for each } g \in G \text {. }
$$

The elements $x_{i}, y_{i}$ are called partial Galois coordinates of $S$ over $R$. By abuse of notation, sometimes we will write $R=S^{\alpha_{G}}$, even when $R$ is some isomorphic copy of $S^{\alpha_{G}}$.

Let $S$ be a partial $\alpha_{G}$-extension of $S^{\alpha_{G}}$. From Theorem 5.1 of [4] we have a correspondence between subgroups of $G$ and certain subalgebras of $S$. Now we give an addendum of this result.

Theorem 3.1. Let $S$ be an algebra, $G$ a finite group, $\alpha_{G}$ a unital partial action of $G$ on $S$ such that $S$ is a partial $\alpha_{G}$-extension of $S^{\alpha_{G}}$ and $H$ a normal subgroup of $G$. Then $\alpha_{G}$ induces a unital partial action $\alpha_{G / H}$ of the quotient group $G / H$ on $S^{\alpha_{H}}$ such that $S^{\alpha_{H}}$ is a partial $\alpha_{G / H^{-}}$extension of $S^{\alpha_{G}}$ and $\left(S^{\alpha_{H}}\right)^{\alpha_{G / H}}=S^{\alpha_{G}}$.

The proof of Theorem 3.1 will be obtained as a consequence of several results which we state and prove below. Let $H$ be a normal subgroup of $G$. By [2, Theorem 2.3], the global action $\beta_{G}$ of $G$ on $T$ induces a global action $\beta_{G / H}$ of $G / H$ on $T^{\beta_{H}}$ in the following way:

$$
\beta_{G / H}: G / H \rightarrow \operatorname{Aut}\left(T^{\beta_{H}}\right),\left.\quad g H \mapsto \beta_{g}\right|_{T^{\beta_{H}}} .
$$

Also, if $T$ is a $G$-extension of $T^{\beta_{G}}$, then $T^{\beta_{H}}$ is a $G / H$-extension of $T^{\beta_{G}}=\left(T^{\beta_{H}}\right)^{\beta_{G / H}}$.

On the other hand, by Proposition 2.1 (iv), $T^{\beta_{H}} e_{H}$ is an ideal of $T^{\alpha_{H}}$. Then the action $\beta_{G / H}$ of $G / H$ on $T^{\beta_{H}}$ induces, by restriction, a partial action $\gamma_{G / H}$ of $G / H$ on $T^{\beta_{H}} e_{H}$, that is, $\gamma_{G / H}=\left(D_{g H}, \gamma_{g H}\right)_{g H \in G / H}$ is given by:

$$
\begin{aligned}
& D_{g H}=\left(T^{\beta_{H}} e_{H}\right) \cap \beta_{g}\left(T^{\beta_{H}} e_{H}\right)=T^{\beta_{H}} e_{g H}, \text { where } e_{g H}:=e_{H} \beta_{g}\left(e_{H}\right), \\
& \gamma_{g H}=\left.\beta_{g}\right|_{D_{g^{-1} H}}: D_{g^{-1} H} \rightarrow D_{g H}, \text { for each } g H \in G / H .
\end{aligned}
$$

Proposition 3.2. The pair $\left(T^{\beta_{H}}, \beta_{G / H}\right)$ is the globalization of the partial action $\gamma_{G / H}$ given in (5) and (6). 
Proof. By construction, (G1), (G2) and (G3) of subsection 2.1 are satisfied. For (G4), it is enough to check that $T^{\beta_{H}}=\sum_{i=1}^{l} \beta_{g_{i}}\left(T^{\beta_{H}} e_{H}\right)$, where $\mathcal{T}=\left\{g_{1}=1, g_{2}, \ldots, g_{l}\right\}$ is a transversal of $H$ on $G$. Let $H=\left\{h_{1}=1, h_{2}, \ldots, h_{m}\right\}$, and write the elements of $G$ in the following order:

$$
G=\left\{1, h_{2}, \ldots, h_{m}, g_{2}, g_{2} h_{2}, \ldots, g_{2} h_{m}, \ldots g_{l}, g_{l} h_{2}, \ldots, g_{l} h_{m}\right\}
$$

We claim that $\beta_{g}\left(T^{\beta_{H}}\right)=T^{\beta_{H}}$, for all $g \in G$. In fact, consider $a \in T^{\beta_{H}}, g \in G$ and $h \in H$. Since $H$ is a normal subgroup of $G$, there is $h^{\prime} \in H$ such that $h g=g h^{\prime}$. Then

$$
\beta_{h}\left(\beta_{g}(a)\right)=\beta_{h g}(a)=\beta_{g h^{\prime}}(a)=\beta_{g}\left(\beta_{h^{\prime}}(a)\right)=\beta_{g}(a) .
$$

Thus $I:=\sum_{i=1}^{l} \beta_{g_{i}}\left(T^{\beta_{H}} e_{H}\right)=\sum_{i=1}^{l} T^{\beta_{H}} \beta_{g_{i}}\left(e_{H}\right)$ is an ideal of $T^{\beta_{H}}$. It is enough to show that $1_{T} \in I$. Take $f_{H}:=\prod_{j=1}^{m}\left(1_{T}-\beta_{h_{j}}\left(1_{S}\right)\right)$. It is clear that $f_{H} \in T^{\beta_{H}}$ and whence $\beta_{g}\left(f_{H}\right) \in T^{\beta_{H}}$, for all $g \in G$. Denote by $e_{i j} \in T$ the idempotent, constructed in (3), associated to the $(m i+j)$-th element of $G$ by considering the order given in (7). Explicitly

$$
e_{i j}=\prod_{1 \leq k \leq i-1} \beta_{g_{k}}\left(f_{H}\right)\left(1_{T}-\beta_{g_{i} h_{1}}\left(1_{S}\right)\right) \cdots\left(1_{T}-\beta_{g_{i} h_{j-1}}\left(1_{S}\right)\right) \beta_{g_{i} h_{j}}\left(1_{S}\right) .
$$

Notice that $e_{1 j}=\left(1_{T}-\beta_{h_{1}}\left(1_{S}\right)\right) \cdots\left(1_{T}-\beta_{h_{j-1}}\left(1_{S}\right)\right) \beta_{h_{j}}\left(1_{S}\right)$. Thus

$$
e_{i j}=\prod_{1 \leq k \leq i-1} \beta_{g_{k}}\left(f_{H}\right) \beta_{g_{i}}\left(e_{1 j}\right) \text {. }
$$

Since $e_{H}=\sum_{j=1}^{m} e_{1 j}$ it follows that $\sum_{j=1}^{m} e_{i j}=\left(\prod_{1 \leq k \leq i-1} \beta_{g_{k}}\left(f_{H}\right)\right) \beta_{g_{i}}\left(e_{H}\right) \in I$. By [4, p.79] we have that $1_{T}=\sum_{\substack{1 \leq i \leq l \\ 1 \leq j \leq m}} e_{i j} \in I$ and consequently $I=T^{\beta_{H}}$.

By Proposition 3.2 we can consider a linear map $\psi_{G / H}: T^{\beta_{H}} \rightarrow T^{\beta_{H}}$ in a similar way as in (2). Indeed, if $G / H=\left\{g_{1} H, \ldots, g_{m} H\right\}$ then

$$
\psi_{G / H}(t)=\sum_{1 \leq l \leq m} \sum_{i_{1}<\cdots<i_{l}}(-1)^{l+1} \beta_{g_{i_{1}}}\left(e_{H}\right) \beta_{g_{i_{2}}}\left(e_{H}\right) \cdots \beta_{g_{i_{l}}}\left(e_{H}\right) \beta_{g_{i_{l}}}(t), \quad t \in T^{\beta_{H}} .
$$

Corollary 3.3. Let $\gamma_{G / H}$ be the partial action of $G / H$ on $T^{\beta_{H}} e_{H}$ given by (5) and (6), $\psi_{G / H}$ the map given by (8) and $R:=\left(T^{\beta_{H}} e_{H}\right)^{\gamma_{G / H}}$ the subalgebra of invariant elements of $T^{\beta_{H}} e_{H}$. Then the following statements hold:

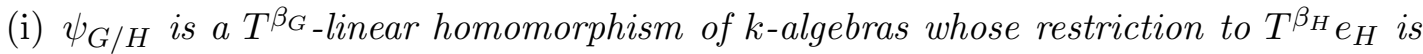
injective and $\psi_{G / H}\left(e_{H}\right)=1_{T}=\psi_{G}\left(1_{S}\right)$.

(ii) $\psi_{G / H}(R) \subset\left(T^{\beta_{H}}\right)^{\beta_{G / H}}=T^{\beta_{G}}$.

(iii) The restriction $\psi_{G / H}$ on $R$ is a k-algebra isomorphism of $R$ onto $T^{\beta_{G}}=\left(T^{\beta_{H}}\right)^{\beta_{G / H}}$ with inverse given by the multiplication by $e_{H}$. Particularly, $T^{\beta_{G}} e_{H}=R$. 
(iv) $T^{\beta_{H}} e_{H}$ is a partial $\gamma_{G / H^{-}}$extension of $R$ if and only if $T^{\beta_{H}}$ is a $\beta_{G / H^{-}}$-xtension of $T^{\beta_{G}}=\left(T^{\beta_{H}}\right)^{\beta_{G / H}}$.

Proof. Items (i), (ii) and (iii) follow directly from Proposition 2.1, while item (iv) follows from Proposition 3.2 and [4, Theorem 3.3].

We shall see that the partial action $\gamma_{G / H}$ on $T^{\beta_{H}} e_{H}$ induces a partial action $\alpha_{G / H}$ of $G / H$ on $S^{\alpha_{H}}$ via multiplication by $1_{S}$. We set

$$
\begin{aligned}
\tilde{1}_{g H} & :=e_{g H} 1_{S}=e_{H} \beta_{g}\left(e_{H}\right) 1_{S}=\beta_{g}\left(e_{H}\right) 1_{S}, \\
\tilde{D}_{g H} & :=D_{g H} 1_{S}=T^{\beta_{H}} e_{g H} 1_{S}=S^{\alpha_{H}} \tilde{1}_{g H}, \\
\alpha_{g H} & :=\left.\left(m_{1_{S}} \circ \gamma_{g H} \circ \psi_{H}\right)\right|_{\tilde{D}_{g^{-1}}},
\end{aligned}
$$

where $m_{1_{S}}: T^{\beta_{H}} e_{H} \rightarrow S^{\alpha_{H}}$ is the multiplication by $1_{S}$. Notice that, for every $s \in S^{\alpha_{H}}$ and $g H \in G / H$,

$$
\alpha_{g H}\left(s \tilde{1}_{g^{-1} H}\right)=\beta_{g}\left(\psi_{H}(s)\right) \tilde{1}_{g H}=\beta_{g}\left(\psi_{H}(s)\right) 1_{S} .
$$

Theorem 3.4. Let $H$ be a normal subgroup of $G$ and $\alpha_{G / H}:=\left(\tilde{D}_{g H}, \alpha_{g H}\right)_{g H \in G / H}$, with $\tilde{D}_{g H}$ and $\alpha_{g H}$ given respectively by (10) and (11). Then the following statements hold:

(i) $\alpha_{G / H}$ is a partial action of $G / H$ on $S^{\alpha_{H}}$.

(ii) $\left(S^{\alpha_{H}}\right)^{\alpha_{G / H}}=S^{\alpha_{G}}$.

(iii) $\left(T^{\beta_{H}}, \beta_{G / H}\right)$ is the globalization of $\alpha_{G / H}$.

(iv) $S^{\alpha_{H}}$ is a partial $\alpha_{G / H^{-}}$extension of $S^{\alpha_{G}}$ if and only if $T^{\beta_{H}}$ is a partial $\beta_{G / H^{-}}$ extension of $T^{\beta_{G}}$.

Proof. (i) Given $g \in G$, it is clear from (9) that $\tilde{1}_{g H}$ is a central idempotent of $T$. We will check that $\tilde{1}_{g H} \in S^{\alpha_{H}}$. Since $H$ is a normal subgroup of $G$, for each $h \in H$, there exists $h^{\prime} \in H$ such that $h g=g h^{\prime}$. Then

$$
\alpha_{h}\left(\tilde{1}_{g H} 1_{h^{-1}}\right)=\alpha_{h}\left(\beta_{g}\left(e_{H}\right) 1_{h^{-1}}\right)=\beta_{h g}\left(e_{H}\right) 1_{h}=\beta_{g h^{\prime}}\left(e_{H}\right) 1_{h} .
$$

By Proposition $2.1(\mathrm{v})$ and (vi), $e_{H}=\psi\left(1_{S}\right) \in T^{\beta_{H}}$. Thus

$$
\alpha_{h}\left(\tilde{1}_{g H} 1_{h^{-1}}\right)=\beta_{g}\left(\beta_{h^{\prime}}\left(e_{H}\right)\right) 1_{h}=\beta_{g}\left(e_{H}\right) 1_{S} 1_{h}=\tilde{1}_{g H} 1_{h} .
$$

Hence $\tilde{D}_{g H}$ is an ideal of $S^{\alpha_{H}}$. Further, by Proposition 2.1,

$$
\psi_{H}\left(S^{\alpha_{H}} \tilde{1}_{g^{-1} H}\right)=\psi_{H}\left(S^{\alpha_{H}}\right) \beta_{g^{-1}}\left(e_{H}\right) e_{H}=T^{\beta_{H}} e_{g^{-1} H} .
$$

Then

$$
\alpha_{g H}\left(\tilde{D}_{g^{-1} H}\right) \stackrel{(13)}{=} m_{1_{S}} \circ \gamma_{g H}\left(T^{\beta_{H}} e_{g^{-1} H}\right)=T^{\beta_{H}} e_{g H} 1_{S}=S^{\alpha_{H}} \beta_{g}\left(e_{H}\right) 1_{S} \stackrel{(10)}{=} \tilde{D}_{g H} .
$$

Thus $\alpha_{g H}: \tilde{D}_{g^{-1} H} \rightarrow \tilde{D}_{g H}$ is a well-defined algebra isomorphism. Hence the condition (P1) of subsection 2.1 is satisfied while (P2) is clear. For (P3), consider $g H, l H \in G / H$ 
and note that

$$
\begin{aligned}
\alpha_{g H}\left(\tilde{D}_{g^{-1} H} \cap \tilde{D}_{l H}\right) & =\left(m_{1_{S}} \circ \gamma_{g H} \circ \psi_{H}\right)\left(S^{\alpha_{H}} \tilde{1}_{g^{-1} H} \tilde{1}_{l H}\right) \\
& \stackrel{(13)}{=}\left(m_{1_{S}} \circ \gamma_{g H}\right)\left(T^{\beta_{H}} e_{g^{-1} H} e_{l H}\right) \stackrel{(*)}{=} m_{1_{S}}\left(T^{\beta_{H}} e_{g H} e_{l g H}\right) \\
& =T^{\beta_{H}} e_{g H} 1_{S} T^{\beta_{H}} e_{l g H} 1_{S}=\tilde{D}_{g_{H}} \cap \tilde{D}_{g l H} .
\end{aligned}
$$

The equality in $(*)$ follows from (P3) because $\gamma_{G / H}$ is a partial action. Finally, to check (P4), take $x \in \tilde{D}_{l^{-1} H} \cap \tilde{D}_{(g l)^{-1} H}$. Then,

$$
\begin{aligned}
\alpha_{g H} \circ \alpha_{l H}(x) & =\left(m_{1_{S}} \circ \gamma_{g H} \circ \psi_{H}\right) \circ\left(m_{1_{S}} \circ \gamma_{h H} \circ \psi_{H}\right)(x) \\
& =\left(m_{1_{S}} \circ\left(\gamma_{g H} \circ \gamma_{l H}\right) \circ \psi_{H}\right)(x) \\
& \stackrel{(13)}{=}\left(m_{1_{S}} \circ \gamma_{g l H} \circ \psi_{H}\right)(x) \\
& =\alpha_{g l H}(x) .
\end{aligned}
$$

(ii) Note that $\tilde{1}_{g^{-1} H} \in S^{\alpha_{H}}$. Since $\psi_{H}$ is left $T^{\beta_{H}}$-linear and $\beta_{g^{-1}}\left(e_{H}\right) \in T^{\beta_{H}}$, it follows that $\psi_{H}\left(\tilde{1}_{g^{-1} H}\right)=\beta_{g^{-1}}\left(e_{H}\right) e_{H}=e_{g^{-1} H}$ (the idempotents $e_{g H}$ are defined in (5)). This implies $\psi_{H}\left(\left(S^{\alpha_{H}}\right)^{\alpha_{G / H}}\right)=\left(\psi_{H}\left(S^{\alpha_{H}}\right)\right)^{\gamma_{G / H}}$. Indeed, let $s \in S^{\alpha_{H}}$ such that $\psi_{H}(s) \in$ $\left(\psi_{H}\left(S^{\alpha_{H}}\right)\right)^{\gamma_{G / H}}$. Then

$$
\begin{aligned}
\alpha_{g H}\left(s \tilde{1}_{g^{-1} H}\right) & \stackrel{(12)}{=} \beta_{g}\left(\psi_{H}(s)\right) \tilde{1}_{g H}=\beta_{g}\left(\psi_{H}(s) e_{g^{-1} H}\right) \tilde{1}_{g H} \\
& =\gamma_{g H}\left(\psi_{H}(s) e_{g^{-1} H}\right) \tilde{1}_{g H}=\psi_{H}(s) e_{g H} 1_{S}=s \tilde{1}_{g H} .
\end{aligned}
$$

In an analogous way one shows the other inclusion. Finally we have

$$
\begin{aligned}
\left(S^{\alpha_{H}}\right)^{\alpha_{G / H}} & \stackrel{(*)}{=} \psi_{H}\left(\left(S^{\alpha_{H}}\right)^{\alpha_{G / H}}\right) 1_{S}=\left(\psi_{H}\left(S^{\alpha_{H}}\right)\right)^{\gamma_{G / H}} 1_{S} \\
& =\left(T^{\beta_{H}} e_{H}\right)^{\gamma_{G / H}} 1_{S} \stackrel{(* *)}{=} T^{\beta_{G}} e_{H} 1_{S} \\
& =T^{\beta_{G}} 1_{S}=S^{\alpha_{G}},
\end{aligned}
$$

where $(*)$ follows from Proposition 2.1 (vii) and $(* *)$ follows from Corollary 3.3 (iii).

(iii) By Proposition 2.1, there exists an algebra monomorphism $\psi_{H}: S^{\alpha_{H}} \rightarrow T^{\beta_{H}}$ such that $\psi_{H}\left(S^{\alpha_{H}}\right)=T^{\beta_{H}} e_{H}$ is an ideal of $T^{\beta_{H}}$, which implies (G1). Furthermore, it follows from (13) that $\psi_{H}\left(\tilde{D}_{g_{H}}\right)=T^{\beta_{H}} e_{g H}=\psi_{H}\left(S^{\alpha_{H}}\right) \cap \beta_{g}\left(\psi_{H}\left(S^{\alpha_{H}}\right)\right)$ and whence (G2) is satisfied. By (12), for each $s \in S^{\alpha_{H}}$,

$$
\begin{aligned}
\psi_{H}\left(\alpha_{g H}\left(s \tilde{1}_{g^{-1} H}\right)\right) & =\psi_{H}\left(\beta_{g}\left(\psi_{H}(s)\right) 1_{S}\right)=\beta_{g}\left(\psi_{H}(s)\right) \psi_{H}\left(1_{S}\right) \\
& =\beta_{g}\left(\psi_{H}(s)\right) e_{H}=\beta_{g}\left(\psi_{H}(s) \tilde{1}_{g^{-1} H}\right) .
\end{aligned}
$$

Thus (G3) is verified. Using Proposition 3.2 we obtain

$$
T^{\beta_{H}}=\sum_{g H \in G / H} \beta_{g H}\left(T^{\beta_{H}} e_{H}\right)=\sum_{g H \in G / H} \beta_{g H}\left(\psi_{H}\left(S^{\alpha_{H}}\right)\right),
$$

and whence (G4) is proved.

(iv) It follows from (iii) and [4, Theorem 3.3]. 
Proof of Theorem 3.1. By Theorem 3.4, there exists a partial action $\alpha_{G / H}$ of the quotient group $G / H$ on $S^{\alpha_{H}}$ induced by $\alpha_{G}$. Observe that by Theorem 2.3 of [2], $T^{\beta_{H}}$ is a $\beta_{G / H^{-}}$ Galois extension of $T^{\beta_{G}}$. Hence, Theorem 3.4 (iv) implies that $S^{\alpha_{H}}$ is an $\alpha_{G / H^{-p a r t i a l}}$ Galois extension of $S^{\alpha}$.

Remark 3.5. Suppose that $\alpha_{G}$ is a global action of $G$ on $S$ and $H$ is a normal subgroup of $G$. Then the $k$-algebra isomorphism $\psi_{H}$ defined in subsection 2.1 is equal to id ${ }_{S}$. Thus, $e_{H}=1_{S}$. Hence the partial action $\alpha_{G / H}$ constructed in (10) and (11) is the usual global action of $G / H$ on $S^{H}$ given by Theorem 2.2 of [2].

In the next proposition we characterize when the partial action $\alpha_{G / H}$ of $G / H$ on $S^{\alpha_{H}}$ given by (10) and (11) is global.

Proposition 3.6. Let $\alpha_{G / H}$ be the partial action of $G / H$ on $S^{\alpha_{H}}$ given by (10) and (11) and assume that $G / H=\left\{g_{1} H=H, g_{2} H, \ldots, g_{m} H\right\}$. Then $\alpha_{G / H}$ is a global action of $G / H$ on $S^{\alpha_{H}}$ if and only if $\left\{\beta_{g_{i}}\left(1_{S}\right): i=1, \ldots, m\right\} \subset \operatorname{ann}\left(1_{T}-e_{H}\right)$.

Proof. Since $\tilde{1}_{g_{i}^{-1} H}=\beta_{g_{i}^{-1}}\left(e_{H}\right) 1_{S}$, we have that $\alpha_{G / H}$ is a global action on $S^{\alpha_{H}}$ if and only if $\beta_{g_{i}^{-1}}\left(e_{H}\right) 1_{S}=1_{S}$ for all $1 \leq i \leq m$. Applying $\beta_{g_{i}}$ in both sides of the last identity we obtain that $e_{H} \beta_{g_{i}}\left(1_{S}\right)=\beta_{g_{i}}\left(1_{S}\right)$ and consequently the result follows.

An immediate consequence of the above proposition is the following.

Corollary 3.7. If $e_{H}=1_{T}$ then the partial action $\alpha_{G / H}$ of $G / H$ on $S^{\alpha_{H}}$ is global.

The next result describes explicitly the idempotents and the isomorphisms given in (9) and (11) respectively. It will be useful in subsection 5.2.

Proposition 3.8. Let $H=\left\{h_{1}=1, h_{2}, \ldots, h_{m}\right\}$ be a normal subgroup of $G, \tilde{1}_{g H}$ given by (9), $\tilde{D}_{g H}$ given by (10) and $\alpha_{g H}$ given by (11). Then

$$
\tilde{1}_{g H}=1_{g}+\sum_{i=2}^{m} \prod_{j=2}^{i}\left(1_{S}-1_{g h_{j-1}}\right) 1_{g h_{i}}
$$

and

$$
\alpha_{g H}(x)=\alpha_{g}\left(x 1_{g^{-1}}\right)+\sum_{i=2}^{m} \prod_{j=1}^{i-1}\left(1_{S}-1_{g h_{j}}\right) \alpha_{g h_{i}}\left(x 1_{\left(g h_{i}\right)^{-1}}\right), \quad x \in \tilde{D}_{g^{-1} H} .
$$

Proof. Since $\tilde{1}_{g H}=\beta_{g}\left(e_{H}\right) 1_{S}$ and $e_{H}=\psi_{H}\left(1_{S}\right)$ we have

$$
\begin{aligned}
\tilde{1}_{g H} & =\sum_{i=1}^{m} \beta_{g h_{i}}\left(1_{S}\right) \beta_{g}\left(e_{i}\right) 1_{S}=\sum_{i=1}^{m} \beta_{g h_{i}}\left(1_{S}\right) 1_{S} \beta_{g}\left(e_{i}\right) 1_{S}=\sum_{i=1}^{m} 1_{g h_{i}} \beta_{g}\left(e_{i}\right) 1_{S} \\
& \stackrel{(4)}{=} 1_{g}+\sum_{i=2}^{m} 1_{g h_{i}} \prod_{j=2}^{i}\left(1_{S}-1_{g h_{j-1}}\right) 1_{g h_{i}}=1_{g}+\sum_{i=2}^{m} \prod_{j=2}^{i}\left(1_{S}-1_{g h_{j-1}}\right) 1_{g h_{i}} .
\end{aligned}
$$


Let $g \in G$ and $x \in \tilde{D}_{g^{-1} H}$. By (11),

$$
\begin{aligned}
\alpha_{g H}(x) & =\left(m_{1_{S}} \circ \gamma_{g H} \circ \psi_{H}\right)(x)=\sum_{i=1}^{m} \beta_{g h_{i}}(x) \beta_{g}\left(e_{i}\right) 1_{S} \\
& =\sum_{i=1}^{m} \alpha_{g h_{i}}\left(x 1_{\left(g h_{i}\right)^{-1}}\right) \beta_{g}\left(e_{i}\right) 1_{S} \\
& =\alpha_{g}\left(x 1_{g^{-1}}\right)+\sum_{i=2}^{m} \alpha_{g h_{i}}\left(x 1_{\left(g h_{i}\right)^{-1}}\right) \prod_{j=2}^{i}\left(1_{S}-1_{g h_{j-1}}\right) . \\
& =\alpha_{g}\left(x 1_{g^{-1}}\right)+\sum_{i=2}^{m} \prod_{j=1}^{i-1}\left(1_{S}-1_{g h_{j}}\right) \alpha_{g h_{i}}\left(x 1_{\left(g h_{i}\right)^{-1}}\right) .
\end{aligned}
$$

We end this section with examples that illustrate the constructions given above.

Example 3.9. Let $R$ be a commutative $k$-algebra and $S:=R e_{1} \oplus R e_{2} \oplus R e_{3}$, where $e_{1}, e_{2}, e_{3}$ are non-zero orthogonal idempotents and $e_{1}+e_{2}+e_{3}=1_{S}$. We consider the partial action $\alpha_{G}$ of the cyclic group of $G=C_{4}=\left\langle g: g^{4}=1\right\rangle$ of order 4 on $S$ given in Example 6.1 of [4]. The ideals are

$$
S_{g}=R e_{1} \oplus R e_{2}, \quad S_{g^{2}}=R e_{1} \oplus R e_{3}, \quad S_{g^{3}}=R e_{2} \oplus R e_{3},
$$

and the isomorphisms are

$$
\alpha_{g}\left(a e_{2}+b e_{3}\right)=a e_{1}+b e_{2}, \quad \alpha_{g^{2}}\left(a e_{1}+b e_{3}\right)=a e_{3}+b e_{1}, \quad \alpha_{g^{3}}\left(a e_{1}+b e_{2}\right)=a e_{2}+b e_{3},
$$

for all $a, b \in R$. Given the subgroup $H=C_{2}=\left\{1, g^{2}\right\}$ of $C_{4}$, note that the quotient group is $G / H=\{H, g H\} \simeq C_{2}$ and $S^{\alpha_{H}}=R\left(e_{1}+e_{3}\right) \oplus R e_{2}$. It is clear that $\left(T, \beta_{G}\right)$ is the globalization of $\alpha_{G}$, where $T=S \oplus R e_{4}$ and $\beta_{g}\left(e_{j}\right)=e_{j-1(\bmod 4)}$. From (2) it follows that

$$
\psi_{H}\left(a e_{1}+b e_{2}+c e_{3}+d e_{4}\right)=a e_{1}+b\left(e_{2}+e_{4}\right)+c e_{3}, \quad \text { for all } a, b, c, d \in R .
$$

Hence, $e_{H}=\psi_{H}\left(1_{S}\right)=1_{T}$ and whence $\tilde{1}_{g H}=\beta_{g}\left(1_{T}\right) 1_{S}=1_{S}$. Thus $\tilde{D}_{g H}=S^{\alpha_{H}}$ and using (12) we conclude that $\alpha_{g H}\left(a\left(e_{1}+e_{3}\right)+b e_{2}\right)=b\left(e_{1}+e_{3}\right)+a e_{2}$. In this case, $G / H$ acts globally on $S^{\alpha_{H}}$.

Example 3.10. [9, Example 4.2] Let $R$ be a commutative $k$-algebra and $T:=\oplus_{i=1}^{6} R e_{i}$ where $\left\{e_{i}\right\}_{i=1}^{6}$ is a set of non-zero orthogonal idempotents whose sum is $1_{T}$. Consider the action $\beta_{G}$ of the cyclic group $G=C_{6}=\left\langle g: g^{6}=1\right\rangle$ of order 6 on $T$ given by

$$
\beta_{g^{j}}\left(\sum_{i=1}^{6} a_{i} e_{i}\right)=\sum_{i=1}^{6} a_{i} e_{i+j(\bmod 6)}, \quad a_{i} \in R .
$$

Let $S:=\operatorname{Re}_{1} \oplus R e_{3} \oplus R e_{6}$ and $\alpha_{G}=\left(S_{g}, \alpha_{g}\right)_{g \in G}$ be the induced partial action of $\beta_{G}$ on $S$. Explicitly

$$
S_{g}=R e_{1}, \quad S_{g^{2}}=R e_{3}, \quad S_{g^{3}}=R e_{3} \oplus R e_{6}, \quad S_{g^{4}}=R e_{1}, \quad S_{g^{5}}=R e_{6},
$$


and $\alpha_{g^{j}}=\beta_{g^{j}}: S_{g^{6-j}} \rightarrow S_{g^{j}}$, for all $1 \leq j \leq 5$. By construction, $\left(T, \beta_{G}\right)$ is the globalization of $\alpha_{G}$. Given the subgroup $H=C_{2}=\left\{1, g^{3}\right\}$ of $C_{6}$, the quotient group is $G / H=\left\{H, g H, g^{2} H\right\} \simeq C_{3}$ and $S^{\alpha_{H}}=R e_{1} \oplus R\left(e_{3}+e_{6}\right)$. From (2) it follows that

$$
\psi_{H}\left(\sum_{i=1}^{6} a_{i} e_{i}\right)=a_{1}\left(e_{1}+e_{4}\right)+a_{3} e_{3}+a_{6} e_{6}, \quad \text { for all } a_{i} \in R, \quad 1 \leq i \leq 6 .
$$

Thus $e_{H}=\psi_{H}\left(1_{S}\right)=e_{1}+e_{3}+e_{4}+e_{6}$ and consequently

$$
\tilde{1}_{H}=1_{S}, \quad \tilde{1}_{g H}=\beta_{g}\left(e_{H}\right) 1_{S}=e_{1}, \quad \tilde{1}_{g^{2} H}=\beta_{g^{2}}\left(e_{H}\right) 1_{S}=e_{3}+e_{6} .
$$

Hence the partial action $\alpha_{G / H}=\left(\tilde{D}_{g}, \alpha_{g H}\right)_{g H \in G / H}$ of $G / H$ on $S^{\alpha_{H}}$ is given by

$$
\tilde{D}_{H}=S^{\alpha_{H}}, \quad \tilde{D}_{g H}=R e_{1}, \quad \tilde{D}_{g^{2} H}=R\left(e_{3}+e_{6}\right),
$$

and

$$
\alpha_{H}=\operatorname{id}_{S^{\alpha} H}, \quad \alpha_{g H}\left(a\left(e_{3}+a_{6}\right)\right)=a e_{1}, \quad \alpha_{g^{2} H}\left(a e_{1}\right)=a\left(e_{3}+e_{6}\right), \quad a \in R .
$$

\section{On $G$-isomorphic partial Galois extensions}

In this section we introduce the notion of $G$-isomorphic partial $\alpha_{G}$-extensions and we investigate its relation with $G$-isomorphic $G$-extensions given in [6].

Let $\alpha_{G}=\left(S_{g}, \alpha_{g}\right)_{g \in G}$ and $\alpha_{G}^{\prime}=\left(S_{g}^{\prime}, \alpha_{g}^{\prime}\right)_{g \in G}$ be partial actions of $G$ on the commutative $k$-algebras $S$ and $S^{\prime}$ respectively. Assume that $S$ (resp. $S^{\prime}$ ) is a partial $\alpha_{G^{-}}$extension (resp. $\alpha_{G}^{\prime}$-extension) of $R \simeq S^{\alpha_{G}}\left(\right.$ resp. $\left.R \simeq\left(S^{\prime}\right)^{\alpha_{G}^{\prime}}\right)$. We say that $\left(S, \alpha_{G}\right)$ and $\left(S^{\prime}, \alpha_{G}^{\prime}\right)$ are partially $G$-isomorphic if there exists a $k$-algebra isomorphism $f: S \rightarrow S^{\prime}$ which is $R$-linear and satisfies

$$
f\left(S_{g}\right) \subseteq S_{g}^{\prime} \quad \text { and }\left.\quad\left(f \circ \alpha_{g}\right)\right|_{S_{g^{-1}}}=\left.\left(\alpha_{g}^{\prime} \circ f\right)\right|_{S_{g^{-1}}}, \quad \text { for all } g \in G .
$$

In this case, we will denote $\left(S, \alpha_{G}\right) \stackrel{p a r}{\sim}\left(S^{\prime}, \alpha_{G}^{\prime}\right)$. Clearly $\stackrel{p a r}{\sim}$ is an equivalence relation on the set of partial $\alpha_{G}$-extensions of $R$. The equivalence class of $\left(S, \alpha_{G}\right)$ will be denoted by $\left\lfloor S, \alpha_{G}\right\rfloor$.

Remark 4.1. Notice that if $f: S \rightarrow S^{\prime}$ is a $k$-algebra isomorphism such that $f\left(S_{g}\right) \subset S_{g}^{\prime}$ then $f\left(S_{g}\right)=S_{g}^{\prime}$. Indeed, since $f$ is surjective, given $a_{g}^{\prime} \in S_{g}^{\prime}$ there exists $a \in S$ such that $f(a)=a_{g}^{\prime}$. Also, $f\left(1_{g}\right)=1_{g}^{\prime}$. Thus, $a_{g}^{\prime}=f(a) 1_{g}^{\prime}=f\left(a 1_{g}\right)$ which implies that $f\left(S_{g}\right)=S_{g}^{\prime}$.

In order to prove the next result we recall from [4] the definition of the trace map. Let $\alpha_{G}=\left(S_{g}, \alpha_{g}\right)_{g \in G}$ be a partial action of $G$ on $S$ and $R=S^{\alpha_{G}}$. The trace map $t r_{\alpha_{G}}: S \rightarrow S$ is defined by

$$
t r_{\alpha_{G}}(s)=\sum_{g \in G} \alpha_{g}\left(s 1_{g^{-1}}\right), \quad s \in S .
$$

By [4, Lemma 2.1], $t r_{\alpha_{G}}(S) \subset R$ and $t r_{\alpha_{G}}$ is a (left and right) $R$-linear map.

Proposition 4.2. Let $\left(S, \alpha_{G}\right)$ (resp. $\left(S^{\prime}, \alpha_{G}^{\prime}\right)$ ) be a partial $\alpha_{G^{-}}$extension (resp. $\alpha_{G^{-}}^{\prime}$ extension) of $R$. Then $\left(S, \alpha_{G}\right)$ and $\left(S^{\prime}, \alpha_{G}^{\prime}\right)$ are partially $G$-isomorphic if and only if there exists a $k$-algebra homomorphism $f: S \rightarrow S^{\prime}$ which is $R$-linear and satisfies (16). 
Proof. Let $\alpha_{G}=\left(S_{g}, \alpha_{g}\right)_{g \in G}$ and $\alpha_{G}^{\prime}=\left(S_{g}^{\prime}, \alpha_{g}^{\prime}\right)_{g \in G}$ be partial actions of $G$ on $S$ and $S^{\prime}$ respectively. Assume that $f: S \rightarrow S^{\prime}$ is a $k$-algebra homomorphism which is $R$-linear and satisfies (16). Since $S$ is a partial $\alpha_{G^{-}}$extension of $R$, consider $x_{i}, y_{i} \in S, 1 \leq i \leq n$, the partial Galois coordinates of $S$ over $R$. For each $s^{\prime} \in S^{\prime}$, we have

$$
\begin{aligned}
f\left(\sum_{i=1}^{n} x_{i} t r_{\alpha^{\prime}}\left(f\left(y_{i} 1_{g^{-1}}\right) s^{\prime}\right)\right) & =\sum_{i=1}^{n} \sum_{g \in G} f\left(x_{i}\right) \alpha_{g}^{\prime}\left(f\left(y_{i} 1_{g^{-1}}\right)\right) \alpha_{g}^{\prime}\left(s^{\prime} 1_{g^{-1}}^{\prime}\right) \\
& =\sum_{i=1}^{n} \sum_{g \in G} f\left(x_{i}\right)\left(\alpha_{g}^{\prime} \circ f\right)\left(y_{i} 1_{g^{-1}}\right) \alpha_{g}^{\prime}\left(s^{\prime} 1_{g^{-1}}^{\prime}\right) \\
& =\sum_{i=1}^{n} \sum_{g \in G} f\left(x_{i}\right)\left(f \circ \alpha_{g}\right)\left(y_{i} 1_{g^{-1}}\right) \alpha_{g}^{\prime}\left(s^{\prime} 1_{g^{-1}}^{\prime}\right) \\
& =\sum_{g \in G} f\left(\sum_{i=1}^{n} x_{i} \alpha_{g}\left(y_{i} 1_{g^{-1}}\right)\right) \alpha_{g}^{\prime}\left(s^{\prime} 1_{g^{-1}}^{\prime}\right) \\
& =f\left(1_{S}\right) s^{\prime}=1_{S^{\prime}} s^{\prime}=s^{\prime},
\end{aligned}
$$

which implies that $f$ is surjective. Suppose that $s \in S$ and $f(s)=0$. Then

$$
\begin{aligned}
f\left(\alpha_{g}\left(y_{i} s 1_{g^{-1}}\right)\right) & =\left(f \circ \alpha_{g}\right)\left(y_{i} s 1_{g^{-1}}\right)=\left(\alpha_{g}^{\prime} \circ f\right)\left(y_{i} s 1_{g^{-1}}\right) \\
& =\alpha_{g}^{\prime}\left(f\left(y_{i}\right) f(s) f\left(1_{g^{-1}}\right)\right)=0,
\end{aligned}
$$

for all $g \in G$. Since $\operatorname{tr}_{\alpha_{G}}(S) \subset R$, we have $\operatorname{tr}_{\alpha_{G}}\left(y_{i} s\right) 1_{S^{\prime}}=f\left(\operatorname{tr}_{\alpha_{G}}\left(y_{i} s\right) 1_{S}\right)$. Thus, $\operatorname{tr}_{\alpha_{G}}\left(y_{i} s\right)=0$, for all $1 \leq i \leq n$. Hence,

$$
\begin{aligned}
0 & =\sum_{i=1}^{n} x_{i} \operatorname{tr}_{\alpha_{G}}\left(y_{i} s\right)=\sum_{g \in G}\left(\sum_{i=1}^{n} x_{i} \alpha_{g}\left(y_{i} 1_{g^{-1}}\right)\right) \alpha_{g}\left(s 1_{g^{-1}}\right) \\
& =\sum_{g \in G} \delta_{1, g} \alpha_{g}\left(s 1_{g^{-1}}\right)=s
\end{aligned}
$$

which proves that $f$ is injective.

Let $\left(T, \beta_{G}\right)$ and $\left(T^{\prime}, \beta_{G}^{\prime}\right)$ be $G$-extensions of $A \simeq T^{\beta_{G}} \simeq\left(T^{\prime}\right)^{\beta_{G}^{\prime}}$. According to [6], $\left(T, \beta_{G}\right)$ and $\left(T^{\prime}, \beta_{G}^{\prime}\right)$ are $G$-isomorphic if there is an $A$-linear homomorphism of $k$-algebras $f: T \rightarrow T^{\prime}$ such that $f \circ \beta_{g}=\beta_{g}^{\prime} \circ f$, for all $g \in G$. In this case, $f$ is in fact an isomorphism; more details can be seen in [6].

For our purposes, the concept of (global) $G$-isomorphism for globalization of partial actions needs one more condition than the classical notion. Let $\left(S, \alpha_{G}\right)$ and $\left(S^{\prime}, \alpha_{G}^{\prime}\right)$ be partial actions of $G$ such that $S$ and $S^{\prime}$ are, respectively, partial $\alpha_{G}$ and $\alpha_{G}^{\prime}$-extensions of $R$. Let $\left(T, \beta_{G}\right)$ and $\left(T^{\prime}, \beta_{G}^{\prime}\right)$ be their respective globalizations. It follows from Theorem 3.3 of [4] that $T$ and $T^{\prime}$ are $G$-extensions of $A \simeq T^{\beta_{G}} \simeq\left(T^{\prime}\right)^{\beta_{G}^{\prime}}$. In this case, we say that $\left(\left(T, \beta_{G}\right),\left(S, \alpha_{G}\right)\right)$ and $\left(\left(T^{\prime}, \beta_{G}^{\prime}\right),\left(S^{\prime}, \alpha_{G}^{\prime}\right)\right)$ are $G$-isomorphic if there exists an $A$-linear $G$-isomorphism $f: T \rightarrow T^{\prime}$ such that $f\left(1_{S}\right)=1_{S^{\prime}}$. We will write $\left(\left(T, \beta_{G}\right),\left(S, \alpha_{G}\right)\right) \sim$ $\left(\left(T^{\prime}, \beta_{G}^{\prime}\right),\left(S^{\prime}, \alpha_{G}^{\prime}\right)\right)$ to indicate that $\left(\left(T, \beta_{G}\right),\left(S, \alpha_{G}\right)\right)$ and $\left(\left(T^{\prime}, \beta_{G}^{\prime}\right),\left(S^{\prime}, \alpha_{G}^{\prime}\right)\right)$ are $G$ isomorphic. Clearly $\sim$ is an equivalence relation. 
Let $\left(S, \alpha_{G}\right)$ be a partial action of $G$ on $S$ and $H$ a normal subgroup of $G$. As in section 3 , here $\left(S^{\alpha_{H}}, \alpha_{G / H}\right)$ denotes the partial action of $G / H$ on $S^{\alpha_{H}}$ given by (10) and (11).

Theorem 4.3. Let $\left(S, \alpha_{G}\right)$ and $\left(S^{\prime}, \alpha_{G}^{\prime}\right)$ be partial actions of $G$ and $\left(T, \beta_{G}\right)$ and $\left(T^{\prime}, \beta_{G}^{\prime}\right)$ their respective globalizations. Assume that $S$ and $S^{\prime}$ are, respectively, partial $\alpha_{G}$ and $\alpha_{G}^{\prime}$-extensions of $R$. Then the following statements are equivalent:

(i) $\left(\left(T, \beta_{G}\right),\left(S, \alpha_{G}\right)\right) \sim\left(\left(T^{\prime}, \beta_{G}^{\prime}\right),\left(S^{\prime}, \alpha_{G}^{\prime}\right)\right)$,

(ii) $\left(S^{\alpha_{H}}, \alpha_{G / H}\right) \stackrel{p a r}{\sim}\left(\left(S^{\prime}\right)^{\alpha_{H}^{\prime}}, \alpha_{G / H}^{\prime}\right)$, for all normal subgroup $H$ of $G$,

(iii) $\left(S, \alpha_{G}\right) \stackrel{\text { par }}{\sim}\left(S^{\prime}, \alpha_{G}^{\prime}\right)$.

Proof. (i) $\Rightarrow$ (ii) We suppose that $H=\left\{h_{1}=1, h_{2}, \ldots, h_{m}\right\}, A \simeq T^{\beta_{G}} \simeq\left(T^{\prime}\right)^{\beta_{G}}$ and $R \simeq S^{\alpha_{G}} \simeq\left(S^{\prime}\right)^{\alpha_{G}^{\prime}}$. Consider an $A$-linear $k$-algebra isomorphism $f: T \rightarrow T^{\prime}$ such that

$$
f\left(1_{S}\right)=1_{S^{\prime}}, \quad f \circ \beta_{g}=\beta_{g}^{\prime} \circ f, \quad \text { for all } g \in G .
$$

It is easy to check that $\left.f\right|_{T^{\beta_{H}}}: T^{\beta_{H}} \rightarrow\left(T^{\prime}\right)^{\beta_{H}^{\prime}}$ is an $A$-linear $k$-algebra isomorphism. By Proposition 3.2, $\left(T^{\beta_{H}}, \beta_{G / H}\right)$ is the globalization of $\left(T^{\beta_{H}} e_{H}, \gamma_{G / H}\right)$, where $\gamma_{G / H}$ is given by (5) and (6). Observe that $e_{H}=\psi_{H}\left(1_{S}\right)=\sum_{j=1}^{m} \beta_{h_{j}}\left(1_{S}\right) e_{j}$, where the family $\left\{e_{i}\right\}_{1 \leq i \leq m}$ is given by (3). Similarly, we have that $\left(\left(T^{\prime}\right)^{\beta_{H}^{\prime}}, \beta_{G / H}^{\prime}\right)$ is the globalization of $\left(\left(T^{\prime}\right)^{\beta_{H}^{\prime}} e_{H}^{\prime}, \gamma_{G / H}^{\prime}\right)$, where $e_{H}^{\prime}=\sum_{j=1}^{m} \beta_{h_{j}}^{\prime}\left(1_{S^{\prime}}\right) e_{j}^{\prime}$ and

$$
e_{1}^{\prime}=1_{S^{\prime}} \text { and } e_{j}^{\prime}=\left(1_{T^{\prime}}-1_{S^{\prime}}\right) \cdots\left(1_{T^{\prime}}-\beta_{h_{j-1}}^{\prime}\left(1_{S^{\prime}}\right)\right) \beta_{h_{j}}^{\prime}\left(1_{S^{\prime}}\right), \quad 1 \leq j \leq m .
$$

It follows from (17) that $f\left(e_{j}\right)=e_{j}^{\prime}$, for all $1 \leq j \leq m$. Thus, $f\left(T^{\beta_{H}} e_{H}\right)=\left(T^{\prime}\right)^{\beta_{H}^{\prime}} e_{H}^{\prime}$. In particular, $f\left(e_{H}\right)=e_{H}^{\prime}$.

We recall the partial actions $\alpha_{G / H}$ and $\alpha_{G / H}^{\prime}$ of $G / H$ on $S^{\alpha_{H}}$ and $\left(S^{\prime}\right)^{\alpha_{H}^{\prime}}$ respectively. They are given by (10) and (11), that is, the ideals are $\tilde{D}_{g H}=T^{\beta_{H}} e_{H} \beta_{g}\left(e_{H}\right) 1_{S}$ and $\tilde{D}_{g H}^{\prime}=\left(T^{\prime}\right)^{\beta_{H}^{\prime}} e_{H}^{\prime} \beta_{g}^{\prime}\left(e_{H}^{\prime}\right) 1_{S^{\prime}}$ and the partial isomorphisms are

$$
\alpha_{g H}=\left.\left(m_{1_{S}} \circ \gamma_{g H} \circ \psi_{H}\right)\right|_{\tilde{D}_{g^{-1} H}}, \quad \alpha_{g H}^{\prime}=\left.\left(m_{1_{S^{\prime}}} \circ \gamma_{g H}^{\prime} \circ \psi_{H}^{\prime}\right)\right|_{\tilde{D}_{g^{\prime} H_{H}}} .
$$

Consider the map $\varphi=m_{1_{S^{\prime}}} \circ f \circ \psi_{H}: S^{\alpha_{H}} \rightarrow\left(S^{\prime}\right)^{\alpha_{H}^{\prime}}$. By Proposition 2.1, $\psi_{H}\left(S^{\alpha_{H}}\right) \subset$ $T^{\beta_{H}}$. Since $f\left(T^{\beta_{H}}\right)=\left(T^{\prime}\right)^{\beta_{H}^{\prime}}$ and $\left(T^{\prime}\right)^{\beta_{H}^{\prime}} 1_{S^{\prime}}=\left(S^{\prime}\right)^{\alpha_{H}^{\prime}}$, it follows that $\varphi$ is well-defined. Clearly, $\varphi$ is a $k$-algebra homomorphism. We will check that $\varphi$ is $R$-linear. By item (vi) of Proposition 2.1 we obtain $S^{\alpha_{G}}=T^{\beta_{G}} 1_{S} \simeq\left(T^{\prime}\right)^{\beta_{G}^{\prime}} 1_{S^{\prime}}=\left(S^{\prime}\right)^{\alpha_{G}^{\prime}}$. Hence, given $a \in T^{\beta_{G}}$ we have that $a 1_{S} \in S^{\alpha_{G}}$. Moreover there exists a unique $a^{\prime} \in\left(T^{\prime}\right)^{\beta_{G}^{\prime}}$ such that $a^{\prime} 1_{S^{\prime}} \in\left(S^{\prime}\right)^{\alpha_{G}^{\prime}}$. Then, for $s \in S^{\alpha_{H}}$,

$$
\begin{aligned}
\varphi\left(\left(a 1_{S}\right) s\right) & =\left(m_{1_{S^{\prime}}} \circ f \circ \psi_{H}\right)\left(\left(a 1_{S}\right) s\right) \stackrel{(*)}{=}\left(m_{1_{S^{\prime}}} \circ f\right)\left(a \psi_{H}\left(1_{S}\right) \psi_{H}(s)\right) \\
& =\left(m_{1_{S^{\prime}}} \circ f\right)\left(a e_{H} \psi_{H}(s)\right)=1_{S^{\prime}}\left(a^{\prime} f\left(e_{H}\right) f\left(\psi_{H}(s)\right)\right) \\
& =1_{S^{\prime}}\left(a^{\prime} e_{H}^{\prime} f\left(\psi_{H}(s)\right)\right)=\left(a^{\prime} 1_{S^{\prime}}\right)\left(1_{S^{\prime}} e_{H}^{\prime}\right) f\left(\psi_{H}(s)\right) \\
& =\left(a^{\prime} 1_{S^{\prime}}\right)\left(1_{S^{\prime}} f\left(\psi_{H}(s)\right)=\left(a^{\prime} 1_{S^{\prime}}\right) \varphi(s) .\right.
\end{aligned}
$$


The equality $(*)$ follows because $\psi_{H}$ is $A$-linear by Proposition 2.1 (ii). Now, we check that $\varphi$ satisfies (16). For each $g \in G$, we have

$$
\begin{aligned}
\varphi\left(\tilde{D}_{g H}\right) & \stackrel{(13)}{=} m_{1_{S^{\prime}}} \circ f\left(T^{\beta_{H}} e_{g H}\right)=\left(m_{1_{S^{\prime}}} \circ f\right)\left(T^{\beta_{H}} \beta_{g}\left(e_{H}\right) e_{H}\right) \\
& =1_{S^{\prime}} T^{\beta_{H}^{\prime}} f\left(\beta_{g}\left(e_{H}\right)\right) f\left(e_{H}\right)=1_{S^{\prime}} T^{\prime \beta_{H}^{\prime}} \beta_{g}^{\prime}\left(f\left(e_{H}\right)\right) e_{H}^{\prime} \\
& =T^{\prime \beta_{H}^{\prime}} e_{H}^{\prime} \beta_{g}^{\prime}\left(e_{H}^{\prime}\right) 1_{S^{\prime}}=\tilde{D}_{g H}^{\prime} .
\end{aligned}
$$

Also, if $x \in \tilde{D}_{g^{-1} H}$ then

$$
\begin{aligned}
& \left(\varphi \circ \alpha_{g H}\right)(x)=\left(m_{1_{S^{\prime}}} \circ\left(f \circ \gamma_{g H}\right) \circ \psi_{H}\right)(x) \\
& \stackrel{(17)}{=}\left(m_{1_{S^{\prime}}} \circ\left(\gamma_{g H}^{\prime} \circ f\right) \circ \psi_{H}\right)(x) \\
& =\left(m_{1_{S^{\prime}}} \circ \gamma_{g H}^{\prime} \circ \operatorname{id}_{\left(T^{\prime}\right)^{H} e_{H}^{\prime}} \circ f \circ \psi_{H}\right)(x) \\
& \stackrel{(*)}{=}\left(\left(m_{1_{S^{\prime}}} \circ \gamma_{g H}^{\prime} \circ\left(\psi_{H}^{\prime} \circ m_{1_{S^{\prime}}}\right) \circ f \circ \psi_{H}\right)\right)(x) \\
& =\left(\left(m_{1_{S^{\prime}}} \circ \gamma_{g H}^{\prime} \circ \psi_{H}^{\prime}\right) \circ\left(m_{1_{S^{\prime}}} \circ f \circ \psi_{H}\right)\right)(x) \\
& =\left(\alpha_{g H}^{\prime} \circ \varphi\right)(x) \text {. }
\end{aligned}
$$

The equality $(*)$ follows from Proposition 2.1 (vi) because $\left(T^{\prime}\right)^{\beta_{H}^{\prime}} e_{H}^{\prime} 1_{S^{\prime}}=\left(S^{\prime}\right)^{\alpha_{H}^{\prime}}$ and $\psi_{H}^{\prime}\left(\left(S^{\prime}\right)^{\alpha_{H}^{\prime}}\right)=\left(T^{\prime}\right)^{\beta_{H}^{\prime}} e_{H}^{\prime}$. By Proposition 4.2, $\left(S^{\alpha_{H}}, \alpha_{G / H}\right)$ and $\left(\left(S^{\prime}\right)^{\alpha_{H}^{\prime}}, \alpha_{G / H}^{\prime}\right)$ are partially $G / H$-isomorphic.

It is clear that (ii) $\Rightarrow$ (iii) follows by taking the trivial subgroup $H=\{1\}$ of $G$. Finally, for (iii) $\Rightarrow$ (i), we consider a $k$-algebra isomorphism $f: S \rightarrow S^{\prime}$ satisfying (16) and an injective homomorphism of $k$-algebras $\varphi^{\prime}: S^{\prime} \rightarrow T^{\prime}$ satisfying (G1)-(G4) of $\S 2.1$. We claim that $\left(T^{\prime}, \beta_{G}^{\prime}\right)$ is a globalization of $\left(S, \alpha_{G}\right)$. In fact, consider the $k$-algebra monomorphism $\phi=\varphi^{\prime} \circ f: S \rightarrow T^{\prime}$. We shall check that $\phi$ also satisfies the conditions (G1)-(G4). Observe that (G1) is immediate. Let $g \in G$. Using Remark 4.1, we have that

$$
\begin{aligned}
\phi\left(S_{g}\right) & =\varphi^{\prime}\left(f\left(S_{g}\right)\right)=\varphi^{\prime}\left(S_{g}^{\prime}\right) \stackrel{(\mathrm{G} 2)}{=} \varphi^{\prime}\left(S^{\prime}\right) \cap \beta_{g}^{\prime}\left(\varphi^{\prime}\left(S^{\prime}\right)\right) \\
& =\left(\varphi^{\prime} \circ f\right)(S) \cap\left(\beta_{g}^{\prime} \circ \varphi^{\prime} \circ f\right)(S)=\phi(S) \cap \beta_{g}^{\prime}(\phi(S)),
\end{aligned}
$$

which implies that $\phi$ satisfies (G2). For (G3), notice that

$$
\left(\phi \circ \alpha_{g}\right)(x)=\left(\varphi^{\prime} \circ f \circ \alpha_{g}\right)(x)=\left(\varphi^{\prime} \circ \alpha_{g}^{\prime} \circ f\right)(x) \stackrel{(\mathrm{G} 3)}{=}\left(\beta_{g}^{\prime} \circ \varphi^{\prime} \circ f\right)(x)=\left(\beta_{g}^{\prime} \circ \phi\right)(x),
$$

for all $g \in G$ and $x \in S_{g^{-1}}$. Finally,

$$
T^{\prime}=\sum_{g \in G} \beta_{g}^{\prime}\left(\varphi^{\prime}\left(S^{\prime}\right)\right)=\sum_{g \in G} \beta_{g}^{\prime}\left(\left(\varphi^{\prime} \circ f\right)(S)\right)=\sum_{g \in G} \beta_{g}^{\prime}(\phi(S)) .
$$

Thus $\left(T^{\prime}, \beta_{G}^{\prime}\right)$ is a globalization of $\left(S, \alpha_{G}\right)$. Since $\left(T, \beta_{G}\right)$ is also a globalization of $\left(S, \alpha_{G}\right)$, by Theorem 4.5 of [3], the global actions $\left(T^{\prime}, \beta_{G}^{\prime}\right)$ and $\left(T, \beta_{G}\right)$ are equivalent. Particularly, $A \simeq T^{\beta_{G}} \simeq\left(T^{\prime}\right)^{\beta_{G}^{\prime}}$. Also, it follows from the proof of Theorem 4.5 of [3] that $\Psi: T \rightarrow T^{\prime}$ given by $\Psi\left(\sum_{g \in G} \beta_{g}(\varphi(s))\right)=\sum_{g \in G} \beta_{g}^{\prime}(\phi(s))$ is an $A$-linear algebra isomorphism such that $\Psi \circ \beta_{g}=\beta_{g}^{\prime} \circ \Psi$, for all $g \in G$, and $\Psi\left(1_{S}\right)=1_{S^{\prime}}$. 
Example 4.4. Let $G=\left\langle g: g^{4}=1\right\rangle$ be the cyclic group of order 4. Consider an algebra $R$ and $T:=R e_{1} \oplus R e_{2} \oplus R e_{3} \oplus R e_{4}$, where $\left\{e_{i}\right\}_{1 \leq i \leq 4}$ is a set of orthogonal idempotents whose sum is $1_{T}$. The group $G$ acts on $T$ by $\beta_{g^{i}}\left(e_{j}\right)=e_{j-i(\bmod 4)}$. Let $e=e_{1}+e_{2}+e_{3} \in T, S=T e$ and $\alpha_{G}$ be the induced partial action of $G$ on $S$, i. e. $\alpha_{G}=\left(S_{g}, \alpha_{g}\right)_{g \in G}$ with $S_{g^{i}}=S \cap \beta_{g^{i}}(S)$ and $\alpha_{g^{i}}=\left.\beta_{g^{i}}\right|_{S_{g^{4-i}}}$. By Example 6.1 of [4], $\left(S, \alpha_{G}\right)$ is a partial $\alpha_{G}$-extension of $R$. Notice that $\left(T, \beta_{G}\right)$ is $G$-isomorphic to $\left(T, \beta_{G}\right)$ but $\left(\left(T, \beta_{G}\right),\left(T, \beta_{G}\right)\right)$ is not $G$-isomorphic to $\left(\left(T, \beta_{G}\right),\left(S, \alpha_{G}\right)\right)$.

\section{The commutative inverse semigroup $\mathrm{T}_{p a r}(G, R)$}

From now on, $G$ will denote a finite abelian group. Given a commutative $k$-algebra $R, \mathrm{~T}_{\text {par }}(G, R)$ denotes the set of equivalence classes of partial abelian $\alpha_{G}$-extensions of $R$, that is, the elements of $\mathrm{T}_{\text {par }}(G, R)$ are the classes $\left\lfloor S, \alpha_{G}\right\rfloor$, where $\left(S, \alpha_{G}\right)$ is a partial action of $G$ on $S, R=S^{\alpha_{G}}$ and $R \subset S$ is a partial abelian $\alpha_{G^{-}}$extension. In this section we define an operation on $\mathrm{T}_{\text {par }}(G, R)$ which turns it into a commutative inverse semigroup. First we recall from [6] the classical construction of the Harrison group.

5.1. The Harrison group. Let $A$ be a commutative $k$-algebra and $\mathrm{T}(G, A)$ the set of the equivalence classes of $G$-isomorphic abelian extensions of $A$. Given a Galois extension $\left(B, \beta_{G}\right)$ of $A$ (which means that $\beta_{G}$ is a global action of $G$ on $B, A \simeq B^{\beta_{G}}$ and $A \subset B$ is an abelian $\beta_{G}$-extension), we will denote its corresponding equivalence class by $\left[B, \beta_{G}\right]$. It was shown in $[6]$ that $\mathrm{T}(G, A)$ is an abelian group and now we recall this construction.

Given $\left[B, \beta_{G}\right],\left[B^{\prime}, \beta_{G}^{\prime}\right] \in \mathrm{T}(G, A)$, the tensor product $B \otimes_{A} B^{\prime}$ is an abelian $\beta_{G \times G^{-}}$ extension of $A$. By Theorem 2.2 of [2], $\left(B \otimes_{A} B^{\prime}\right)^{\delta G}$ is an abelian $\beta_{G \simeq(G \times G) / \delta G^{-e x t e n s i o n}}$ of $A$, where $\delta G=\left\{\left(g, g^{-1}\right): g \in G\right\}$. The group $G$ acts on $\left(B \otimes_{A} B^{\prime}\right)^{\delta G}$ via the group homomorphism $\gamma_{G}: G \rightarrow$ Aut $\left(\left(B \otimes_{A} B^{\prime}\right)^{\delta G}\right)$ given by

$$
\gamma_{g}\left(\sum_{i=1}^{l} b_{i} \otimes b_{i}^{\prime}\right)=\sum_{i=1}^{l} \beta_{g}\left(b_{i}\right) \otimes b_{i}^{\prime}=\sum_{i=1}^{l} b_{i} \otimes \beta_{g}^{\prime}\left(b_{i}^{\prime}\right), \quad b_{i} \in B, \quad b_{i}^{\prime} \in B^{\prime} .
$$

Consequently

$$
\left[B, \beta_{G}\right] *\left[B^{\prime}, \beta_{G}^{\prime}\right]=\left[\left(B \otimes_{A} B^{\prime}\right)^{\delta G}, \gamma_{(G \times G) / \delta G}\right]
$$

defines an associative and commutative operation on $\mathrm{T}(G, A)$. The identity element of $\mathrm{T}(G, A)$ is the class $\left[E_{G}(A), \rho_{G}\right]$ which is defined in the following way. Consider symbols $\left\{e_{g}\right\}_{g \in G}$ and the free $A$-module $E_{G}(A)=\oplus_{g \in G} A e_{g}$ with basis $\left\{e_{g}\right\}_{g \in G}$. The product on $E_{G}(A)$ is defined by $\left(a e_{g}\right)\left(a^{\prime} e_{h}\right)=\delta_{g, h}\left(a a^{\prime}\right) e_{g}$, for all $a, a^{\prime} \in A$ and $g, h \in G$. Then $E_{G}(A)$ is a $k$-algebra with unity $1_{E_{G}(A)}=\sum_{g \in G} e_{g}$. The action $\rho_{G}$ of $G$ on $E_{G}(A)$ is given by $\rho_{g}\left(a e_{h}\right)=a e_{g h}$, for all $a \in A$ and $g, h \in G$. The inverse of $\left[B, \beta_{G}\right]$ is the equivalence class $\left[B, \beta_{G}^{-1}\right]$, where the action $\beta_{G}^{-1}$ of $G$ on $B$ is defined by $\beta_{g}^{-1}(b):=\beta_{g^{-1}}(b)$, for all $g \in G$ and $b \in B$.

The group $\mathrm{T}(G, A)$ is called Harrison group and its study reduces to the case $G$ cyclic; see [6] for more details. 
5.2. The construction of $\mathrm{T}_{\text {par }}(G, R)$. We recall that a semigroup $\mathcal{I}$ is regular if for each $x \in \mathcal{I}$, there exists $x^{*} \in \mathcal{I}$ such that $x x^{*} x=x$ and $x^{*} x x^{*}=x^{*}$. A regular semigroup $\mathcal{I}$ is said an inverse semigroup if the idempotents of $\mathcal{I}$ commute with each other. Then, commutative regular semigroups are inverse semigroups. It is known that a semigroup $\mathcal{I}$ is inverse if and only if for each $x \in \mathcal{I}$ there exists a unique $x^{*} \in \mathcal{I}$ such that $x x^{*} x=x$ and $x^{*} x x^{*}=x^{*}$. The element $x^{*}$ is called the inverse of $x$. The set of idempotents of $\mathcal{I}$ is $E(\mathcal{I})=\left\{x^{*} x: x \in \mathcal{I}\right\}$. The set $E(\mathcal{I})$ admits a partial order via: $e \leq f$ if $e f=e$, where $e, f \in E(\mathcal{I})$. It is well-known that $E(\mathcal{I})$ is a meet semilattice with respect to the partial order $\leq$. We suggest [7] and [8] for more details on regular and inverse semigroups.

In order to construct a commutative inverse semigroup, denote by $\mathrm{T}_{p a r}(G, R)$ the set of all equivalence class $\left\lfloor S, \alpha_{G}\right\rfloor$, where $\left(S, \alpha_{G}\right)$ is a partial action of $G$ on $S, R \simeq S^{\alpha_{G}}$ and $R \subset S$ is a partial abelian $\alpha_{G^{-}}$extension. Consider $\left\lfloor S, \alpha_{G}\right\rfloor,\left\lfloor S^{\prime}, \alpha_{G}^{\prime}\right\rfloor \in \mathrm{T}_{\text {par }}(G, R)$.

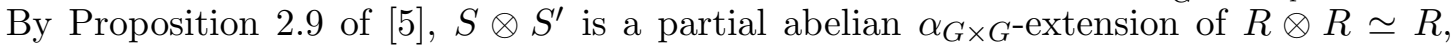
where $\alpha_{G \times G}:=\alpha_{G} \otimes \alpha_{G}^{\prime}$. We define on $\mathrm{T}_{\text {par }}(G, R)$ the operation $*_{\text {par }}$ by

$$
\left\lfloor S, \alpha_{G}\right\rfloor *_{p a r}\left\lfloor S^{\prime}, \alpha_{G}^{\prime}\right\rfloor=\left\lfloor\left(S \otimes S^{\prime}\right)^{\alpha_{\delta G}}, \alpha_{(G \times G) / \delta G}\right\rfloor,
$$

where $\delta G=\left\{\left(g, g^{-1}\right): g \in G\right\}$ and $\alpha_{(G \times G) / \delta G}$ is given by (10) and (11).

Now we will prove the main result of this paper.

Theorem 5.1. $\left(\mathrm{T}_{\text {par }}(G, R), *_{\text {par }}\right)$ is a commutative inverse semigroup.

Proof. Suppose that $\left(S_{1}, \alpha_{G, 1}\right) \stackrel{p a r}{\sim}\left(S_{1}^{\prime}, \alpha_{G, 1}^{\prime}\right)$ and $\left(S_{2}, \alpha_{G, 2}\right) \stackrel{p a r}{\sim}\left(S_{2}^{\prime}, \alpha_{G, 2}^{\prime}\right)$. Then, it is clear that $\left(S_{1} \otimes S_{2}, \alpha_{G, 1} \otimes \alpha_{G, 2}\right) \stackrel{p a r}{\sim}\left(S_{1}^{\prime} \otimes S_{2}^{\prime}, \alpha_{G, 1}^{\prime} \otimes \alpha_{G, 2}^{\prime}\right)$. Using the implication (iii) $\Rightarrow$ (ii) of Theorem 4.3 for the subgroup $\delta G$ of $G \times G$, we conclude that * par is welldefined. Observe that from Theorem 3.1 it follows that $\mathrm{T}_{\text {par }} G, R$ ) is closed under such a product. It is clear that $*_{\text {par }}$ is an associative and commutative operation. Thus it is enough to check that $\mathrm{T}_{\text {par }}(G, R)$ is regular. Let $\left\lfloor S, \alpha_{G}\right\rfloor$ be an element of $\mathrm{T}_{\text {par }}(G, R)$. Assume that $\left(T, \beta_{G}\right)$ is the globalization of $\left(S, \alpha_{G}\right)$ and $T^{\beta_{G}}=A$. Consider the partial action $\alpha_{G}^{*}=\left(S_{g}^{*}, \alpha_{g}^{*}\right)_{g \in G}$ of $G$ on $S$ defined by $S_{g}^{*}:=S_{g^{-1}}$ and $\alpha_{g}^{*}:=\alpha_{g^{-1}}$, for all $g \in \mathcal{G}$. Thus, the global action $\left(T, \beta_{G}^{-1}\right)$ of $G$ on $T$ given by $\beta_{g}^{-1}:=\beta_{g^{-1}}$ for each $g \in G$, is the globalization of $\left(S, \alpha_{G}^{*}\right)$. Since $\left[T, \beta_{G}\right] *\left[T, \beta_{G}\right]^{-1}=\left[E_{G}(A), \rho_{G}\right]$ in $T(G, A)$, it follows that $\left[T, \beta_{G}\right] *\left[T, \beta_{G}^{-1}\right] *\left[T, \beta_{G}\right]=\left[T, \beta_{G}\right]$.

Denote $T^{\prime}:=\left(T \otimes_{A} T\right)^{\delta G}$ and $T^{\prime \prime}:=\left(T^{\prime} \otimes_{A} T\right)^{\delta G}$. Then

$$
\begin{aligned}
\left(\left[T, \beta_{G}\right] *\left[T, \beta_{G}^{-1}\right]\right) *\left[T, \beta_{G}\right] & =\left[T^{\prime}, \gamma_{G}^{\prime}\right] *\left[T, \beta_{G}\right] \\
& =\left[T^{\prime \prime}, \gamma_{G}^{\prime \prime}\right]
\end{aligned}
$$

where $\gamma_{G}^{\prime}, \gamma_{G}^{\prime \prime}$ are given by (18) and $\delta G=\left\{\left(g, g^{-1}\right): g \in G\right\}$. Also, the map

$$
f: T^{\prime \prime} \rightarrow T, \quad f((x \otimes y) \otimes z)=x y z,
$$


is a $k$-algebra homomorphism which is $A$-linear and $f\left(\left(1_{S} \otimes 1_{S}\right) \otimes 1_{S}\right)=1_{S}$. Note that $\beta_{g} \circ f=f \circ \gamma_{g}^{\prime \prime}$, for all $g \in G$. Indeed, consider $t=\left(t_{1} \otimes t_{2}\right) \otimes t_{3} \in T^{\prime \prime}$. Then

$$
\begin{aligned}
\gamma_{g}^{\prime \prime}(t) & \stackrel{(18)}{=}\left(t_{1} \otimes t_{2}\right) \otimes \beta_{g}\left(t_{3}\right)=\left(t_{1} \otimes \beta_{g^{-1}}\left(\beta_{g}\left(t_{2}\right)\right)\right) \otimes \beta_{g}\left(t_{3}\right) \\
& \stackrel{(*)}{=}\left(\beta_{g}\left(t_{1}\right) \otimes \beta_{g}\left(t_{2}\right)\right) \otimes \beta_{g}\left(t_{3}\right),
\end{aligned}
$$

where $(*)$ follows because $t_{1} \otimes t_{2} \in T^{\prime}$. Thus, $\left(\beta_{g} \circ f\right)(t)=\left(f \circ \gamma_{g}^{\prime \prime}\right)(t)$. Since the maps $f, \beta_{g}, \gamma_{g}^{\prime \prime}$ are $A$-linear, we conclude that $\beta_{g} \circ f=f \circ \gamma_{g}^{\prime \prime}$. Then, by Proposition $4.2, f$ is an isomorphism. By Theorem 3.4, $\left(T^{\prime \prime}, \gamma_{G}^{\prime \prime}\right)$ is the globalization of $\left(S^{\prime \prime}, \alpha_{G}^{\prime \prime}\right)$ where

$$
S^{\prime \prime}=\left(S^{\prime} \otimes S\right)^{\alpha_{\delta G}^{\prime \prime}}, \quad S^{\prime}=(S \otimes S)^{\alpha_{\delta G}^{\prime}}, \quad \alpha_{G}^{\prime \prime}=\alpha_{G^{\prime}}^{\prime} \otimes \alpha_{G}, \quad \alpha_{G}^{\prime}=\alpha_{G} \otimes \alpha_{G}^{*},
$$

and $G^{\prime}=(G \times G) / \delta G \simeq G$. Thus $\left(\left(T^{\prime \prime}, \gamma_{G}^{\prime \prime}\right),\left(S^{\prime \prime}, \alpha_{G}^{\prime \prime}\right) \sim\left(\left(T, \beta_{G}\right),\left(S, \alpha_{G}\right)\right)\right.$. It follows from Theorem 4.3 that $\left(S^{\prime \prime}, \alpha_{G}^{\prime \prime}\right) \stackrel{p a r}{\sim}\left(S, \alpha_{G}\right)$, that is, $\left\lfloor S, \alpha_{G}\right\rfloor *_{\text {par }}\left\lfloor S, \alpha_{G}^{*}\right\rfloor *_{\text {par }}\left\lfloor S, \alpha_{G}\right\rfloor=$ $\left\lfloor S, \alpha_{G}\right\rfloor$. In a similar way one shows that $\left\lfloor S, \alpha_{G}^{*}\right\rfloor *_{\text {par }}\left\lfloor S, \alpha_{G}\right\rfloor *_{\text {par }}\left\lfloor S, \alpha_{G}^{*}\right\rfloor=\left\lfloor S, \alpha_{G}^{*}\right\rfloor$. Consequently $\left\lfloor S, \alpha_{G}^{*}\right\rfloor=\left\lfloor S, \alpha_{G}\right\rfloor^{*}$ and $\mathrm{T}_{\text {par }}(G, R)$ is a regular semigroup.

In the next example we calculate explicitly an idempotent of $\mathrm{S}(G, R)$.

Example 5.2. Let $G=\left\langle g: g^{4}=1\right\rangle$ be the cyclic group of order 4. Consider an algebra $R$ and $S:=R e_{1} \oplus R e_{2}$, where $e_{1}, e_{2}$ are non-zero orthogonal idempotents whose sum is one. Here we calculate the idempotent of $\mathrm{T}_{\text {par }}(G, R)$ associated to an easy partial action of $G$ on $S$. We define the partial action $\theta_{G}$ of $G$ on $S$ by taking $S_{1}=S, S_{g}=R e_{2}$, $S_{g^{2}}=\{0\}, S_{g^{3}}=R e_{1}$, and setting $\theta_{1}=\operatorname{id}_{S}, \theta_{g}\left(r e_{1}\right)=r e_{2}, \theta_{g^{2}}=0, \theta_{g^{3}}\left(r e_{2}\right)=r e_{1}$, $r \in R$. Clearly $S^{\theta_{G}}=R$. Also, $x_{1}=y_{1}=e_{1}$ and $x_{2}=y_{2}=e_{2}$ are partial Galois coordinates of $S$ over $R$. We will calculate the idempotent $\left\lfloor S, \theta_{G}\right\rfloor{ }^{*} *_{p a r}\left\lfloor S, \theta_{G}\right\rfloor$. Denote by $e_{i j}:=e_{i} \otimes e_{j}$ and $e_{i 0}=e_{i} \otimes 1_{S}$. It is straightforward to check that

$$
\tilde{S}:=(S \otimes S)^{\delta G}=R\left(e_{11}+e_{22}\right) \oplus R e_{12} \oplus R e_{21},
$$

where $\theta_{G}^{*}$ is the partial action of $G$ on $S$ defined by $\theta_{g}^{*}=\theta_{g^{-1}}$, for all $g \in G$. Now we calculate the idempotents $1_{(l, 1) \delta_{G}}$, with $l \in G$. Let $h_{i}=g^{i-1}, 1 \leq i \leq 4$. By (14),

$$
1_{(l, 1) \delta G}=1_{l^{-1}} \otimes 1_{S}+\sum_{i=2}^{4} \prod_{j=1}^{i-1}\left(1_{S} \otimes 1_{S}-1_{\left(l h_{j}\right)^{-1}} \otimes 1_{h_{j}^{-1}}\right)\left(1_{\left(l h_{i}\right)^{-1}} \otimes 1_{h_{i}^{-1}}\right) .
$$

Consequently

$\tilde{1}_{(1,1) \delta G}=1_{S} \otimes 1_{S}, \quad \tilde{1}_{(g, 1) \delta G}=e_{10}+e_{22}, \quad \tilde{1}_{\left(g^{2}, 1\right) \delta G}=e_{12}+e_{21}, \quad \tilde{1}_{\left(g^{3}, 1\right) \delta G}=e_{20}+e_{11}$.

It is immediate to verify that

$$
\begin{gathered}
\tilde{D}_{(g, 1) \delta G}=R\left(e_{11}+e_{22}\right) \oplus R e_{12}, \quad \tilde{D}_{\left(g^{2}, 1\right) \delta G}=R e_{12} \oplus R e_{21}, \\
\tilde{D}_{\left(g^{3}, 1\right) \delta G}=R\left(e_{11}+e_{22}\right) \oplus R e_{21} .
\end{gathered}
$$

It follows from (15) that $\left\lfloor S, \theta_{G}\right\rfloor^{*} *_{\text {par }}\left\lfloor S, \theta_{G}\right\rfloor=\left\lfloor\tilde{S}, \tilde{\theta_{G}}\right\rfloor$, where

$$
\begin{gathered}
\tilde{\theta}_{g}\left(r\left(e_{11}+e_{22}\right) \oplus s e_{21}\right)=r\left(e_{11}+e_{22}\right)+s e_{12}, \\
\tilde{\theta}_{g^{2}}\left(r e_{12}+s e_{21}\right)=s e_{12}+r e_{21}, \quad \tilde{\theta}_{g^{3}}=\tilde{\theta}_{g}^{-1}, \quad r, s \in R .
\end{gathered}
$$


5.3. A group isomorphism. Let $\varphi: \mathcal{I} \rightarrow \mathcal{J}$ be a homomorphism of semigroups. We recall from subsection 2.3 of [8] that the kernel of $\varphi$ is the equivalence relation on $\mathcal{I}$ defined by

$$
\operatorname{ker} \varphi:=\{(x, y) \in \mathcal{I} \times \mathcal{I}: \varphi(x)=\varphi(y)\}
$$

In fact, $\rho:=\operatorname{ker} \varphi$ is a congruence on $\mathcal{I}$. Denote by $\rho(a)$ an equivalence class on $S$ and by $\mathcal{I} / \rho$ the set of all equivalence classes. Thus $\mathcal{I} / \rho$ has the following natural structure of semigroup: $\rho(a) \rho(b)=\rho(a b)$, for all $a, b \in S$. This operation is well-defined because $\rho$ is a congruence. If $\varphi$ is surjective then $\mathcal{I} / \rho \simeq \mathcal{J}$ (as semigroups).

Let $R$ be an algebra and $\left\lfloor S, \alpha_{G}\right\rfloor \in \mathrm{T}_{\text {par }}(G, R)$. Consider a globalization $\left(T, \beta_{G}\right)$ of $\left(S, \alpha_{G}\right)$. By Theorem 3.3 of [4], $T$ is an abelian $\beta_{G}$-extension of $T^{\beta_{G}} \simeq A$, that is, $\left[T, \beta_{G}\right] \in T(G, A)$. Moreover, if $\left(S, \alpha_{G}\right),\left(S^{\prime}, \alpha_{G}^{\prime}\right)$ are, respectively, partial abelian $\alpha_{G}$ and $\alpha_{G^{-}}^{\prime}$-extensions of $R$ and $\left(T, \beta_{G}\right),\left(T^{\prime}, \beta_{G}^{\prime}\right)$ are their respective globalizations, then we have $T^{\beta_{G}} \simeq\left(T^{\prime}\right)^{\beta_{G}^{\prime}}$. Hence, we can define the following map

$$
\pi: \mathrm{T}_{p a r}(G, R) \rightarrow \mathrm{T}(G, A), \quad \pi\left(\left\lfloor S, \alpha_{G}\right\rfloor\right)=\left[T, \beta_{G}\right] .
$$

By Theorem 4.3, $\pi$ is well-defined. It is clear that $\pi$ is a surjective homomorphism of semigroups. Hence, we have the following result.

Theorem 5.3. Let $R$ be an algebra and $\rho=\operatorname{ker} \pi$, where $\pi$ is given by (21). Then

$$
\bar{\pi}: T_{p a r}(G, R) / \rho \rightarrow T(G, A), \quad \bar{\pi}\left(\rho\left(\left\lfloor S, \alpha_{G}\right\rfloor\right)\right)=\left[T, \beta_{G}\right],
$$

is a group isomorphism.

5.4. Reduction to cyclic groups. As in the classical case, the study of partial Galois extensions of finite abelian groups reduces to cyclic groups. In fact, let $G$ be a finite abelian group and assume that $G=G_{1} \times G_{2} \times \cdots \times G_{n}$, where $G_{1}, G_{2}, \ldots, G_{n}$ are cyclic groups. For each $1 \leq i \leq n$, let $\left(S_{i}, \alpha_{G_{i}}\right)$ be a partial action of $G_{i}$ on $S_{i}, R=S_{i}^{\alpha_{G_{i}}}$ and assume that $R \subset S_{i}$ is a partial abelian $\alpha_{G_{i}}$-extension. Consider $S:=S_{1} \otimes S_{2} \otimes \cdots \otimes S_{n}$ and $\alpha_{G}:=\alpha_{G_{1}} \otimes \alpha_{G_{2}} \otimes \cdots \otimes \alpha_{G_{n}}$. By Proposition 2.9 of [5], $\left(S, \alpha_{G}\right)$ is a partial action and $S$ is a partial abelian $\alpha_{G}$-extension of $R$. Thus, we have a semigroup homomorphism

$$
\phi: \prod_{i=i}^{n} T_{p a r}\left(G_{i}, R\right) \rightarrow T_{p a r}(G, R), \quad \phi\left(\left\lfloor S_{1}, \alpha_{G_{1}}\right\rfloor,\left\lfloor S_{2}, \alpha_{G_{2}}\right\rfloor, \ldots,\left\lfloor S_{n}, \alpha_{G_{n}}\right\rfloor\right)=\left\lfloor S, \alpha_{G}\right\rfloor .
$$

To construct the inverse of $\phi$, let $\left(S, \alpha_{G}\right)$ be a partial action of $G$ on $S, R=S^{\alpha_{G}}$ and assume that $R \subset S$ is a partial abelian $\alpha_{G}$-extension. For each $1 \leq i \leq n$, we consider the subgroup $H_{i}=G_{1} \times G_{2} \times \cdots \times G_{i-1} \times\{1\} \times G_{i+1} \times \cdots \times G_{n}$ of $G$. Note that $H_{i}$ acts partially on $S$ via restriction. Fix $S_{i}:=S^{\alpha_{H_{i}}}$ and observe that $G / H_{i} \simeq G_{i}$. By Theorem 3.4, $R \subset S_{i}$ is a partial abelian $\alpha_{G_{i}}$-extension. Thus, the inverse of $\phi$ is

$$
\varphi: \mathrm{T}_{p a r}(G, R) \rightarrow \prod_{i=i}^{n} \mathrm{~T}_{p a r}\left(G_{i}, R\right), \quad \varphi\left(\left\lfloor S, \alpha_{G}\right\rfloor\right)=\left(\left\lfloor S_{1}, \alpha_{G_{1}}\right\rfloor,\left\lfloor S_{2}, \alpha_{G_{2}}\right\rfloor, \ldots,\left\lfloor S_{n}, \alpha_{G_{n}}\right\rfloor\right) .
$$




\section{On the Structure of $\mathrm{T}_{\text {par }}(G, R)$}

It is well-known that a commutative inverse semigroup is a strong semilattice of abelian groups; see for instance Corollary IV.2.2 of [7]. Let $R$ be an algebra. By Theorem 5.1,

$$
\mathrm{T}_{p a r}(G, R)=\bigcup_{\xi \in \Lambda} \mathrm{T}_{\xi}(G, R),
$$

with $\Lambda$ a semillatice isomorphic to the set of idempotents of $\mathrm{T}_{p a r}(G, R)$ and $\mathrm{T}_{\xi}(G, R)$ a group, for all $\xi \in \Lambda$. In order to describe the decomposition of $\mathrm{T}_{p a r}(G, R)$ given in (22), we will investigate the idempotents of $\mathrm{T}_{\text {par }}(G, R)$ in the next subsection.

6.1. Idempotents of $\mathrm{T}_{\text {par }}(G, R)$. We recall that the idempotents of $\mathrm{T}_{\text {par }}(G, R)$ are given by $\left\lfloor S, \alpha_{G}\right\rfloor^{*} *_{\text {par }}\left\lfloor S, \alpha_{G}\right\rfloor$, with $\left\lfloor S, \alpha_{G}\right\rfloor \in \mathrm{T}_{\text {par }}(G, R)$. In order to characterize this elements we introduce some extra notation. For the unital partial action $\alpha_{G}=$ $\left(S_{g}, \alpha_{g}\right)_{g \in G}$ of $G$ on $S$ we define:

$$
\begin{aligned}
& \hat{S}:=\prod_{g \in G} S_{g}, \quad \hat{S}_{(h, l)}=\prod_{g \in G} S_{g} 1_{h} 1_{l^{-1}}, \quad \text { for all }(h, l) \in G \times G, \\
& \gamma_{(h, l)}\left(\left(a_{g} 1_{h^{-1}} 1_{l g}\right)_{g \in G}\right)=\left(\alpha_{h}\left(a_{g} 1_{h^{-1}}\right) 1_{h l g}\right)_{g \in G}, \quad \text { for all }\left(a_{g}\right)_{g \in G} \in \hat{S} .
\end{aligned}
$$

As in the previous section, $\alpha_{G}^{*}$ denotes the partial action of $G$ on $S$ given by $\alpha_{g}^{*}=\alpha_{g^{-1}}$, for all $g \in G$.

Proposition 6.1. Let $\alpha_{G}=\left(S_{g}, \alpha_{g}\right)_{g \in G}$ be a unital partial action of $G$ on $S$ such that $R=S^{\alpha_{G}} \subset S$ is a partial $\alpha_{G}$-extension. Then the following statements hold:

(i) The family of pairs $\gamma_{G \times G}=\left(\hat{S}_{(h, l)}, \gamma_{(h, l)}\right)_{(h, l) \in G \times G}$ given by (23) and (24) is a unital partial action of $G \times G$ on $\hat{S}$.

(ii) The partial action $\theta_{G \times G}:=\alpha_{G}^{*} \otimes \alpha_{G}$ of $G \times G$ on $S \otimes S$ is partially $(G \times G)$ isomorphic to $\gamma$.

Proof. (i) Let $(h, l) \in G \times G$ and $\left(a_{g}\right)_{g \in G} \in \hat{S}$. Notice that

$$
\begin{aligned}
\gamma_{(h, l)}\left(\left(a_{g} 1_{h^{-1}} 1_{l g}\right)_{g \in G}\right) & =\left(\left(\alpha_{h}\left(a_{g} 1_{h^{-1}}\right) 1_{h l g}\right) 1_{h} 1_{h g}\right)_{g \in G} \\
\stackrel{t}{ } & \stackrel{h l g}{=}\left(\left(\alpha_{h}\left(a_{g} 1_{l^{-1}}\right) 1_{t}\right) 1_{h} 1_{l^{-1}}\right)_{t \in G},
\end{aligned}
$$

which implies that $\gamma_{(h, l)}$ is well-defined. Clearly, $\gamma_{(h, l)}$ is an algebra isomorphism whose inverse is $\gamma_{\left(h^{-1}, l^{-1}\right)}$. It is straightforward to check that $\gamma$ is a partial action.

(ii) Consider $\varphi: S \otimes S \rightarrow \hat{S}$ defined by $\varphi(x \otimes y)=\left(x \alpha_{g}\left(y 1_{g^{-1}}\right)\right)_{g \in G}$, for all $x, y \in S$. By Theorem 4.1 (iv) of [4], $\varphi$ is an algebra isomorphism and it is $R$-linear. Given $(h, l) \in G \times G$, we have

$$
\varphi\left(S_{h} \otimes S_{l^{-1}}\right)=\prod_{g \in G} S_{h} \alpha_{g}\left(S_{l^{-1}} 1_{g^{-1}}\right)=\prod_{g \in G} S_{g} S_{h} S_{g l^{-1}}=\hat{S}_{(h, l)} .
$$


Also, if $x, y \in S$ then

$$
\begin{aligned}
\left(\varphi \circ \theta_{(h, l)}\right)\left(x 1_{h^{-1}} \otimes y 1_{l}\right) & =\varphi\left(a f_{h}\left(x 1_{h^{-1}}\right) \otimes \alpha_{l^{-1}}\left(y 1_{l}\right)\right) \\
& =\left(\alpha_{h}\left(x 1_{h^{-1}}\right) \alpha_{g l^{-1}}\left(y 1_{g^{-1} l}\right) 1_{g}\right)_{g \in G} \\
& \stackrel{(*)}{=}\left(\alpha_{h}\left(x 1_{h^{-1}}\right) \alpha_{h t}\left(y 1_{h^{-1} t^{-1}}\right) 1_{h l t}\right)_{t \in G} \\
& =\left(\alpha_{h}\left(x \alpha_{t}\left(y 1_{t^{-1}}\right) 1_{l t} 1_{h^{-1}}\right)\right)_{t \in G},
\end{aligned}
$$

where $(*)$ follows by taking $t=h^{-1} l^{-1} g$. On the other hand

$$
\begin{aligned}
\left(\gamma_{(h, l)} \circ \varphi\right)\left(x 1_{h^{-1}} \otimes y 1_{l}\right) & =\hat{\alpha}_{(h, l)}\left(\left(x 1_{h^{-1}} \alpha_{g}\left(y 1_{g^{-1}}\right) 1_{g l}\right)_{g \in G}\right) \\
& =\gamma_{(h, l)}\left(\left(x \alpha_{g}\left(y 1_{g^{-1}}\right) 1_{h^{-1}} 1_{g l}\right)_{g \in G}\right) \\
& =\left(\alpha_{h}\left(x \alpha_{g}\left(y 1_{g^{-1}}\right) 1_{h^{-1}} 1_{g l}\right)\right)_{g \in G} .
\end{aligned}
$$

Thus $\varphi \circ \theta_{(h, l)}=\gamma_{(h, l)} \circ \varphi$ in $S_{h^{-1}} \otimes S_{l}$, for all $l, t \in G$. Hence $\theta_{G \times G}$ and $\gamma_{G \times G}$ are partially $(G \times G)$-isomorphic.

Let $\alpha_{G}=\left(S_{g}, \alpha_{g}\right)_{g \in G}$ be a partial action of $G$ on $S$ and $\left(\hat{S}, \gamma_{G \times G}\right)$ the partial action given by $(23)$ and $(24)$. Denote by $E\left(S, \alpha_{G}\right):=(\hat{S})^{\gamma_{\delta G}}$ the subalgebra of invariants of $\hat{S}$ under $\gamma_{\delta G}$. As in the previous sections, $\tilde{\gamma}_{G}$ is the partial action of $G \simeq(G \times G) / \delta G$ on $E\left(S, \alpha_{G}\right)$. In the next proposition we will prove that the classes $\left\lfloor E\left(S, \alpha_{G}\right), \tilde{\gamma}_{G}\right\rfloor$, where $\left\lfloor S, \alpha_{G}\right\rfloor \in \mathrm{T}_{\text {par }}(G, R)$, are the idempotents of $\mathrm{T}_{\text {par }}(G, R)$ and they will be characterized.

Proposition 6.2. Let $R$ be an algebra. The idempotents of $\mathrm{T}_{\text {par }}(G, R)$ are the classes $\left\lfloor E\left(S, \alpha_{G}\right), \tilde{\gamma}_{G}\right\rfloor$, where $\left\lfloor\left(S, \alpha_{G}\right)\right\rfloor \in \mathrm{T}_{\text {par }}(G, R)$. Moreover, if $G=\left\{1=g_{1}, g_{2}, \cdots, g_{m}\right\}$ and $\alpha_{G}=\left(S_{g}, \alpha_{g}\right)_{g \in G}$ then

$$
\begin{aligned}
& E\left(S, \alpha_{G}\right)=\left\{\left(a_{g}\right)_{g \in G} \in \hat{S}: \alpha_{h}\left(a_{g} 1_{h^{-1}}\right) 1_{g}=a_{g} 1_{h} 1_{h g}, \text { for all } h \in G\right\} \\
& \tilde{\gamma}_{h}\left(\left(a_{g}\right)_{g \in G}\right)=\left(a_{g} 1_{h g}\right)_{g \in G}+\sum_{i=2}^{m} \prod_{j=1}^{i-1}\left(1_{g}-1_{h g_{j}} 1_{g_{j} g}\right)_{g \in G}\left(a_{g} 1_{g_{i}} 1_{g_{i}} 1_{h g}\right)_{g \in G},
\end{aligned}
$$

for all $h \in G$ and $\left(a_{g}\right)_{g \in G} \in E\left(S, \alpha_{G}\right) \tilde{1}_{\left(h^{-1}, 1\right) \delta G}$, where $\tilde{1}_{\left(h^{-1}, 1\right) \delta G}$ is given by $(14)$.

Proof. By Proposition 6.1, $\left(S \otimes S, \theta_{G \times G}\right)$ and $\left(\hat{S}, \gamma_{G \times G}\right)$ are partially $G \times G$-isomorphic. Hence, Theorem 4.3 implies that $\left((S \otimes S)^{\theta_{\delta G}}, \theta_{G \times G / \delta G}\right)$ and $\left(E\left(S, \alpha_{G}\right), \tilde{\gamma}_{G}\right)$ are partially $G$-isomorphic. Thus, $\left\lfloor E\left(S, \alpha_{G}\right), \tilde{\gamma}_{G}\right\rfloor=\left\lfloor(S \otimes S)^{\theta_{\delta G}}, \theta_{G \times G / \delta G}\right\rfloor=\left\lfloor S, \alpha_{G}\right\rfloor^{*} *_{\text {par }}\left\lfloor S, \alpha_{G}\right\rfloor$ are the idempotents of $\mathrm{T}_{p a r}(G, R)$. Notice that $\left(a_{g}\right)_{g \in G} \in E\left(S, \alpha_{G}\right)$ if and only if

$$
\left(a_{g} 1_{h} 1_{h g}\right)_{g \in G}=\gamma_{\left(h, h^{-1}\right.}\left(\left(a_{g} 1_{h^{-1}} 1_{h^{-1} g}\right)_{g \in G}\right) \stackrel{(24)}{=}\left(\alpha_{h}\left(a_{g} 1_{h^{-1}}\right) 1_{g}\right)_{g \in G}, \text { for all } h \in G
$$


By $(23), \hat{S}_{(h, l)}=\hat{S} \hat{1}_{(h, l)}$, where $\hat{1}_{(h, l)}=\left(1_{g} 1_{h} 1_{l^{-1} g}\right)_{g \in G}$ for all $h, l \in G$. Consider $\mathbf{a}=\left(a_{g}\right)_{g \in G} \in E(S, \alpha) \tilde{1}_{\left(h^{-1}, 1\right) \delta G}$. By (15) and (24), we have

$$
\begin{aligned}
\tilde{\gamma}_{h}(\mathbf{a}) & =\gamma_{(1, h)}\left(\mathbf{a} \hat{1}_{\left(1, h^{-1}\right)}\right)+\sum_{i=2}^{m} \prod_{j=1}^{i-1}\left(\left(1_{g}\right)_{g \in G}-\hat{1}_{\left(g_{j}, h g_{j}^{-1}\right)}\right) \gamma_{\left(g_{i}, h g_{i}^{-1}\right)}\left(\mathbf{a}_{\left(g_{i}^{-1}, h^{-1} g_{i}\right)}\right) \\
& \left.=\left(a_{g} 1_{h g}\right)_{g \in G}+\sum_{i=2}^{m} \prod_{j=1}^{i-1}\left(\left(1_{g}\right)_{g \in G}-\left(1_{g_{j}} 1_{h^{-1} g_{j} g}\right)_{g \in G}\right)\left(\alpha_{g_{i}}\left(a_{g} 1_{g_{i}^{-1}}\right) 1_{h g}\right)_{g \in G}\right) \\
& =\left(a_{g} 1_{h g}\right)_{g \in G}+\sum_{i=2}^{m} \prod_{j=1}^{i-1}\left(\left(1_{g}\right)_{g \in G}-\left(1_{h g_{j}} 1_{g_{j} g}\right)_{g \in G}\right)\left(a_{g} 1_{g_{i}} 1_{g_{i} g} 1_{h g}\right)_{g \in G} \\
& =\left(a_{g} 1_{h g}\right)_{g \in G}+\sum_{i=2}^{m} \prod_{j=1}^{i-1}\left(1_{g}-1_{h g_{j}} 1_{g_{j} g}\right)_{g \in G}\left(a_{g} 1_{g_{i}} 1_{g_{i} g} 1_{h g}\right)_{g \in G} .
\end{aligned}
$$

In order to simplify the notation, denote by $I\left(S, \alpha_{G}\right)$ the idempotent $\left\lfloor E\left(S, \alpha_{G}\right), \tilde{\gamma}\right\rfloor$ of $T_{\text {par }}(G, R)$ associated to $\left\lfloor S, \alpha_{G}\right\rfloor$. By Proposition 6.2 and $(22)$ we have that

$$
\mathrm{T}_{p a r}(G, R)=\bigcup_{I\left(S, \alpha_{G}\right)} T_{I\left(S, \alpha_{G}\right)}(G, R) .
$$

Observe that $\mathrm{T}_{I\left(S, \alpha_{G}\right)}(G, R)$ is a group with identity element $I\left(S, \alpha_{G}\right)$. Moreover

$$
\left\lfloor S^{\prime}, \alpha_{I\left(S, \alpha_{G}\right)}^{\prime}\right\rfloor \in T_{I(S, \alpha)}(G, R) \Longleftrightarrow I\left(S^{\prime}, \alpha_{G}^{\prime}\right)=I\left(S, \alpha_{G}\right) .
$$

Proposition 6.3. Let $\left\lfloor S, \alpha_{G}\right\rfloor,\left\lfloor S^{\prime}, \alpha_{G}^{\prime}\right\rfloor \in \mathrm{T}_{\text {par }}(G, R)$. Then $\left\lfloor S^{\prime}, \alpha_{G}^{\prime}\right\rfloor \in \mathrm{T}_{I\left(S, \alpha_{G}\right)}(G, R)$ if and only if there exist an algebra homomorphism $f: I\left(S, \alpha_{G}\right) \rightarrow I\left(S^{\prime}, \alpha_{G}^{\prime}\right)$ which is $R$-linear and such that $f\left(\left(1_{g}\right)_{g \in G}\right)=\left(1_{g}^{\prime}\right)_{g \in G}$.

Proof. Suppose that $f: I\left(S, \alpha_{G}\right) \rightarrow I\left(S^{\prime}, \alpha_{G}^{\prime}\right)$ is an algebra homomorphism $R$-linear such that $f\left(\left(1_{g}\right)_{g \in G}\right)=\left(1_{g}^{\prime}\right)_{g \in G}$. Then, (14) implies that $f\left(\tilde{1}_{(1, g) \delta G}\right)=\tilde{1}_{(1, g) \delta G}$ and whence $f\left(I\left(S, \alpha_{G}\right) \tilde{1}_{(1, g) \delta G}\right) \subseteq I\left(S^{\prime}, \alpha_{G}^{\prime}\right) \tilde{1}_{(1, g) \delta G}$, for all $g \in G$. It is immediate from Proposition 6.2 that $f$ satisfies the other condition of (16) and consequently the result follows from Proposition 4.2. The converse is immediate.

In the next result we present sufficient conditions for $E\left(S, \alpha_{G}\right)$ to be a (global) $\alpha_{G^{-}}$ extension of $R$.

Proposition 6.4. Let $\left(S, \alpha_{G}\right)$ be a unital partial action of $G$ on $S$. If $\operatorname{ann}_{R}\left(1_{g}\right)=0$ for all $g \in G$, then $E\left(S, \alpha_{G}\right)$ is a (global) $\alpha_{G}$-extension of $R$. In this case, we have that $\left\lfloor S, \alpha_{G}\right\rfloor \in T_{I\left(E_{G}(R), \rho_{G}\right)}(G, R)$.

Proof. For each $g \in G$, consider the surjective algebra homomorphism $\pi_{g}: \hat{S} \rightarrow S_{g}$, given by $\pi_{g}\left(\left(a_{g}\right)\right)=a_{g}$. Denote by $E_{g}\left(S, \alpha_{G}\right):=\pi_{g}\left(E\left(S, \alpha_{G}\right)\right)$. Since $E\left(S, \alpha_{G}\right)$ is a partial $\alpha_{G}$-extension of $R$, it follows from Theorem 4.1 of [4] that $E\left(S, \alpha_{G}\right)$ is a finitely generated projective $R$-module. It is clear that $E_{g}\left(S, \alpha_{G}\right)$ is a finitely generated $R$ module. Also, from $E\left(S, \alpha_{G}\right)=\prod_{g \in G} E_{g}\left(S, \alpha_{G}\right)$ it follows that $E_{g}\left(S, \alpha_{G}\right)$ is a projective 
$R$-module. Note that the map $\iota_{g}: R \rightarrow E_{g}\left(S, \alpha_{G}\right)$, defined by $\iota_{g}(r)=r 1_{g}$ for all $r \in R$, is a monomorphism of algebras. Thus, $\operatorname{rank}_{R_{\wp}} E_{g}\left(S, \alpha_{G}\right)_{\wp} \geq 1$, for any prime ideal $\wp$ of $R$. Hence

$$
|G| \leq \sum_{g \in G} \operatorname{rank}_{R_{\wp}} E_{g}\left(S, \alpha_{G}\right)_{\wp}=\operatorname{rank}_{R_{\wp}} E\left(S, \alpha_{G}\right)_{\wp} .
$$

By Corollary 4.6 of [4], $\operatorname{rank}_{R_{\wp}} E\left(S, \alpha_{G}\right)_{\wp} \leq|G|$ and whence $\operatorname{rank}_{R_{\wp}} E\left(S, \alpha_{G}\right)_{\wp}=|G|$, for any prime ideal $\wp$ of $R$. Using Corollary 4.6 of [4] again, we conclude that $E\left(S, \alpha_{G}\right)$ is a global $\alpha_{G}$-extension of $R$. The last assertion of the proposition is obvious.

Remark 6.5. Let $\left[E_{G}(R), \rho_{G}\right]$ be the identity element of $\mathrm{T}(G, R)$ given in subsection 5.1. It is clear that $\mathrm{T}(G, R)$ is a subgroup of $\mathrm{T}_{I\left(E_{G}(R), \rho_{G}\right)}(G, R)$. In general, it is not true that $\mathrm{T}(G, R)=\mathrm{T}_{I\left(E_{G}(R), \rho_{G}\right)}(G, R)$. In fact, let $\left(S, \alpha_{G}\right)$ be the partial action of $G=C_{4}$ on $S=R e_{1} \oplus R e_{2} \oplus R e_{3}$ given in Example 3.9. It is immediate to check that $S$ is a partial $\alpha_{G}$-extension of $R$ and $\operatorname{ann}_{R}\left(1_{g}\right)=0$, for all $g \in G$. By Proposition 6.4, $\left\lfloor S, \alpha_{G}\right\rfloor \in \mathrm{T}_{I\left(E_{G}(R), \rho_{G}\right)}(G, R)$. However, $\left\lfloor S, \alpha_{G}\right\rfloor \notin \mathrm{T}(G, R)$ because $\left(S, \alpha_{G}\right)$ is not global.

\section{REFERENCES}

[1] M. Auslander and O. Goldman, The Brauer group of a commutative ring, Trans. Amer. Math. Soc. 97 (1960), 367-408.

[2] S. U. Chase, D. K. Harrison, A. Rosenberg, Galois theory and Galois cohomology of comutative rings, Mem. Amer. Math. Soc. 52 (1968), 1-19.

[3] M. Dokuchaev and R. Exel, Associativity of crossed products by partial actions, enveloping actions and partial representations, Trans. Amer. Math. Soc. 357 (2005), 1931-1952.

[4] M. Dokuchaev, M. Ferrero and A. Paques, Partial actions and Galois theory, J. Pure Appl. Algebra 208 (2007), 77-87.

[5] M. Dokuchaev, A. Paques and H. Pinedo, Partial Galois cohomology and related homomorphism, Quart. J. Math. 70 (2019), 737-766.

[6] D. K. Harrison, Abelian extensions of commutative rings, Mem. Amer. Math. Soc. 52 (1965), 1-14.

[7] J. M. Howie, An introduction to semigroup theory, Academic Press, London, NY, 1976.

[8] M. V. Lawson, Inverse semigroups: the theory of partial symmetries, World Scientific, 1998.

[9] A. Paques, V. Rodrigues and A. Sant'Ana, Galois correspondences for partial Galois Azumaya extensions, J. Algebra Appl., 10 (5) (2011), 835-847.

Departamento de Matemática, Universidade Federal de Santa Maria, 97105-900, Santa MARIA, RS, BRAZIL

E-mail address: bagio@smail.ufsm.br

Escuela de Matematicas, Universidad Industrial de Santander, Cra. 27 Calle 9 UiS Edificio 45, Bucaramanga, Colombia

E-mail address: ansecape@correo.uis.edu.co

Departamento de Matemáticas y Estadística, Universidad del Tolima, Santa Helena, Ibagué, Colombia

E-mail address: vemarinc@ut.edu.co

Instituto de Matemática e Estatística, Universidade Federal do Rio Grande do Sul, 91509-900, Porto Alegre-RS, Brazil

E-mail address: paques@mat.ufrgs.br

Escuela de Matematicas, Universidad Industrial de Santander, Cra. 27 Calle 9 UiS Edificio 45, Bucaramanga, Colombia

E-mail address: hpinedot@uis.edu.co 